\title{
Deformation and relaxation of an incompressible viscoelastic body with surface viscoelasticity
}

\author{
Liping Liu ${ }^{\mathrm{a}, \mathrm{b}}$, Miao $\mathrm{Yu}^{\mathrm{b}}$, Hao Lin ${ }^{\mathrm{b}}$, Ramsey Foty ${ }^{\mathrm{c}}$ \\ ${ }^{a}$ Department of Mathematics, Rutgers University, NJ 08854, USA \\ ${ }^{b}$ Department of Mechanical Aerospace Engineering, Rutgers University, NJ 08854, USA \\ ${ }^{c}$ Department of Surgery, Robert Wood Johnson Medical School, Rutgers University, NJ 08854, USA
}

\begin{abstract}
Measuring mechanical properties of cells or cell aggregates has proven to be an involved process due to their geometrical and structural complexity. Past measurements are based on material models that completely neglect the elasticity of either the surface membrane or the interior bulk. In this work, we consider general material models to account for both surface and bulk viscoelasticity. The boundary value problems are formulated for deformations and relaxations of a closed viscoelastic surface coupled with viscoelastic media inside and outside of the surface. The linearized surface elasticity models are derived for the constant surface tension model and the Helfrich-Canham bending model for coupling with the bulk viscoelasticity. For quasi-spherical surfaces, explicit solutions are obtained for the deformation, stress-strain and relaxation behaviors under a variety of loading conditions. These solutions can be applied to extract the intrinsic surface and bulk viscoelastic properties of biological cells or cell aggregates in the indentation, electro-deformation and relaxation experiments.
\end{abstract}

Keywords: Biomechanics, viscoelasticity of cells, relaxation, indentation

\section{Introduction}

It is well-known that the mechanical properties of cells and tissues have important implications in cellular structure and function (Manning et al., 2010; Sun et al., 2012; Bi et al., 2015), the pathogenesis of many diseases (Park et al., 2010; Moeendarbary and Harris, 2014; Suresh, 2007), and can be used to quantify the efficacy of drugs for preventing cancer cell dispersal (Foty, 2013; Shannon et al., 2015). Accordingly, the study of mechanical properties of cells, cell aggregates, tissues and organisms has attracted much attention in recent years. In particular, a number of experimental techniques have been developed for measuring the mechanical behaviors of biological systems ranging from the constituent membrane (Diz-Muñoz et al., 2013; Yu et al., 2015), the F-actin and cytoskeleton (Semmrich et al., 2007; Mofrad, 2009; Fletcher and Mullins, 2010; Stricker et al., 2010), the cytoplasm Moeendarbary et al. (2013) to single cells (Dao et al., 2003; Suresh, 2007; Kollmannsberger and Fabry, 2011),

\footnotetext{
${ }^{*}$ Corresponding author

Email address: liu.liping@rutgers.edu (Liping Liu)
} 
extracellular matrix (Nam et al., 2016), cell aggregates, and tissues (Forgacs et al., 1998; Sugimura et al., 2016). To interpret the experimental results and achieve accurate extraction of mechanical properties of the system, two theoretical components are of fundamental importance: (i) simple but realistic material models for the mechanical behaviors of the system, and (ii) analytical or numerical solutions characterizing the experimental procedures. For instance, in the AFM studies a cell is modeled as a homogeneous 3D elastic medium and Hertzian contact law was used for calculating the "Young's modulus" of the medium (A-Hassan et al., 1998; Rosenbluth et al., 2006; Sokolov, 2007). In micropipette aspiration measurements (Hochmuth, 2000), the cell is either modelled as a 2D "solid" membrane with nontrivial shear modulus (Dao et al., 2003; Park et al., 2010), or a 2D fluid membrane in tension or with bending resistence (Chien et al., 1978; Sokolov, 1989), or a 3D elastic halfspace (Theret et al., 1988). More sophisticated models, e.g., power law models (Bausch et al., 1999), soft glassy rheology (Angelini et al., 2011), purified gel models (Gardel et al., 2003, 2004) and poroelasticity (Moeendarbary et al., 2013) have also been proposed with the aim of interpreting mechanical measurements of cells in a more systematic and unifying manner (Fabry et al., 2001; Kollmannsberger and Fabry, 2011).

In this work we focus on a particular type of experimental setup: a quasi-spherical body (a single cell or cell aggregate) that is deformed by an indenter or an electric field, and then released (Foty, 2015; Yu et al., 2015). In the deformation and relaxation process, the evolution of the shape of the body is recorded by a high-speed camera from which one can obtain the relations between time, applied force, and shape of the body. Upon fitting these relationships with the predictions of theoretical models, we can extract the material properties such as stiffness and viscosity of the constituent surface and bulk materials (Yu et al., 2015).

Unlike conventional materials in physics and engineering, the material models for biological systems are still in development; the current consensus may be that cells of different types or under different conditions shall be described by different material models (Diz-Muñoz et al., 2013). As a complex system, cells or cell aggregates possess mechanical properties that depend on the history and current state of deformation or stress, on the chemical environment of the system in which they are tested, and are in general nonlinear and nonlocal. Though more sophicated nonlinear rate-dependent (presumably more accurate) material models could be employed for present study, we have restricted ourselves to linearized viscoelastic models in pursuit of analytical closed-form solutions so that experimental results can be conveniently used to fit with (semi)-analytical solutions and uncover material properties. As compared with material models used for AFM or micropipette aspiration measurements, a noteworthy advancement lies in that the viscoelasticity of both 3D body and 2D surface and their coupling are taken into account in our model. We believe this general consideration is necessary whenever the bulk modulus is comparable with the surface modulus divided by the size of the body: $\mu_{b} \sim \gamma / R$ ( $\mu_{b}$-bulk modulus, $\gamma$ - surface modulus, e.g., surface tension, $R$ - size). From this viewpoint, prior studies of red blood cells (RBCs) neglect bulk elasticity because RBCs lack a 3D cytoskeleton and hence $\mu_{b} \ll \gamma / R$ (Evans and Needham, 1987; Sleep et al., 1999; Popescu et al., 2006; Park et al., 2010). Meanwhile, measurements of prostate cancer and other cells by AFM (A-Hassan et al., 1998; Ren et al., 2015) and magnetic twisting (Wang et al., 1993; Fabry et al., 2001) are based on the theory of 3D elasticity, assuming that surface elasticity is negligible $\left(\gamma / R \ll \mu_{b}\right)$. For cell aggregates, 
there is convincing experimental and structural evidence that the surface (i.e., boundary cells) plays a pivotal role in determining the mechanical behaviors of the aggregates (Foty et al., 1994; Manning et al., 2010; Foty, 2015). On the other hand, completely neglecting bulk contribution to short-time transient behaviors intuitively seems to be unreasonable, motivating the present general model in which the 2D surface and 3D bulk viscoelasticity are coupled. Moreover, even when restricted to 2D surface elasticity, there are several alternative material models that can be considered: (i) constant surface tension model (Popescu et al., 2006); (ii) Helfrich-Canham bending model (Helfrich, 1973); (iii) Gurtin-Murdoch surface elasticity model (Gurtin and Murdoch, 1975; Park et al., 2010). All of these models have been used to describe the elastic behaviors of (different types of) cells or cell aggregates (Bao and Suresh, 2003; Popescu et al., 2006). For generality, we herein establish a framework inclusive of all these elastic models for 2D membranes.

To achieve explicit closed-form solutions, we assume that the body remains in a quasispherical state during deformation and relaxation. This assumption is realistic for cells in suspensions or free-floating cell aggregates (e.g., produced via the hanging-drop approach) based on experimental observations. Since the deformed body is close to a sphere, we can legitimately linearize the general bulk and surface models with reference to a spherical configuration. The consistent linearization of surface elasticity models turns out to be quite subtle for fluid membrane models (Seifert, 1997), e.g., the surface tension model and HelfrichCanham bending model. Further, we consider the boundary value problem describing the processes of deformation and relaxation. By virtue of the spherical reference configuration, we obtain explicit solutions in terms of vector spherical harmonics which may appear to be a standard exercise in physics and engineering. Nevertheless, the explicit calculations of surface differentials of spherical harmonics are rather technical; details of these calculations are listed in Appendix A for the reader's convenience.

Another technical advancement lies in the consideration of tangential displacements for the viscoelasticity of fluid membranes. For the equilibrium configuration or stability analysis of a homogeneous fluid membrane, consideration of the tangential displacement is unnecessary since it corresponds to a reparametrization of the surface (Givli et al., 2012). However, it is essential to account for tangential displacements if, e.g., (i) the bulk elasticity or viscosity is nontrivial and coupled with surface elasticity (due to the continuity of displacement or velocity), (ii) the surface viscosity is not negligible (Arroyo and DeSimone, 2009), or (iii) the surface has inhomogeneities (Walani and Agrawal, 2015). Therefore, unlike the relaxation processes considered in Seifert (1999), we have to consider the tangential displacement in the linearized surface elasticity models and the balances of linear momentum and energy.

Once material models for the bulk and surface are specified, we formulate the boundary value problems associated with two experiments: (i) the relaxation of the body in an ambient fluid and (ii) the deformation of the body by axis-symmetric indenters. The explicit solutions provide a way to process the experimental data and extract useful relations between material properties. Examples of experimental results are also briefly described to substantiate the applications of the explicit analytical solutions.

The paper is organized as follows. We begin with material models for the interior and exterior bulk in Section 2.1 and the surface membrane in Section 2.2. In particular, the linearization of elastic models for fluid membranes is systematically addressed in Section 2.2. We formulate balance laws for the bulk and surface in Section 3. In Section 4 we present 
general solutions and applications. Specifically, we calculate the explicit solutions for general loading conditions in Section 4.1, apply the solutions to relaxation processes in two exemplary experiments and extract relations between relevant material properties in Section 4.2, and present numerical solutions to the force-indentation depth relation for two types of indenters in Section 4.3. We summarize and present an outlook of future applications in Section 5.

Notation. We employ direct notation for brevity if possible. Vectors are denoted by bold symbols such as $\mathbf{e}, \mathbf{u}$, etc. When index notations are in use, the convention of summation over repeated index is followed. The inner (or dot) product of two vectors $\mathbf{a}, \mathbf{b} \in \mathbb{R}^{3}$ is defined as $\langle\mathbf{a}, \mathbf{b}\rangle \equiv \mathbf{a} \cdot \mathbf{b}:=(\mathbf{a})_{i}(\mathbf{b})_{i}$ whereas the inner (or dot) product between matrices $\mathbf{A}$ and $\mathbf{B}$ of the same size is defined as $\mathbf{A} \cdot \mathbf{B}:=\operatorname{Tr}\left(\mathbf{A}^{T} \mathbf{B}\right)=(\mathbf{A})_{i j}(\mathbf{B})_{i j}$. From the viewpoint of matrices, the $i^{\text {th }}$ row vector of the gradient of a vector field, e.g., $\nabla \mathbf{u}$, is the gradient of the $i^{\text {th }}$ component of $\mathbf{u}$ whereas the "div" operates on the row vectors of a matrix field. Therefore, $\operatorname{div} \nabla \mathbf{u}=\Delta \mathbf{u}$ and $\operatorname{div}\left[(\nabla \mathbf{u})^{T}\right]=\nabla(\operatorname{div} \mathbf{u})$. For a scaling parameter $\varepsilon \ll 1, O(\varepsilon)$ implies the asymptotic behavior $O(\varepsilon) / \varepsilon \rightarrow C \neq 0$ as $\varepsilon \rightarrow 0$ whereas $o(\varepsilon) / \varepsilon \rightarrow 0$ as $\varepsilon \rightarrow 0$.

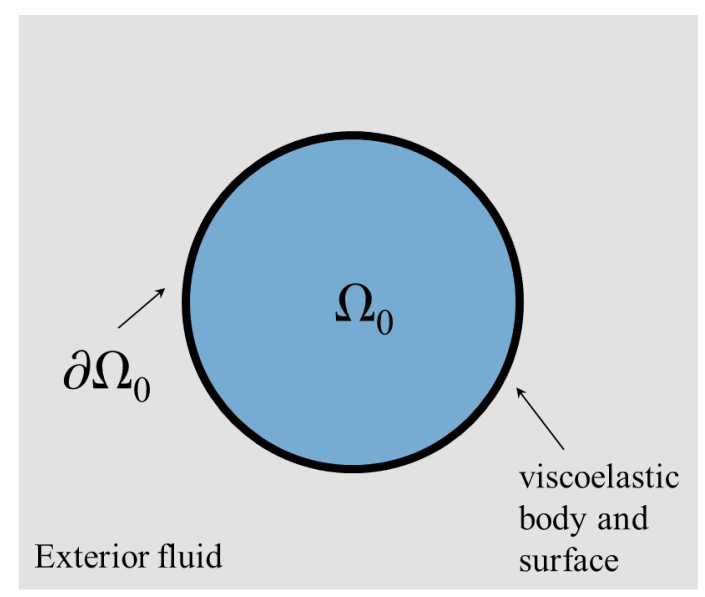

Figure 1: Material models: the system consists of three components: an exterior viscous fluid, a viscoelastic interior bulk and a viscoelastic surface membrane.

\section{Material models}

We first describe the material models for the system. As illustrated in Fig. 1, the system consists of a bulk viscoelastic body encapsulated in a viscoelastic membrane and immersed in a viscous fluid. Let $\Omega_{0} \subset \mathbb{R}^{3}$ be the reference configuration occupied by the overall viscoelastic body. The motion (or state) of the system is represented by the displacement $\mathbf{u}(\mathbf{x}, t): \mathbb{R}^{3} \times[0,+\infty) \rightarrow \mathbb{R}^{3}$. Within the regime of small strain and strain rate, we postulate the following material models for the bulk and surface.

\subsection{Bulk viscoelasticity}

Elasticity of the interior bulk body is modelled as an incompressible elastic solid. In general, the reference configuration $\Omega_{0}$ does not have to be the stress-free equilibrium state. Instead, there can be some eigenstrain (or residue strain) $\epsilon_{0}: \Omega_{0} \rightarrow \mathbb{R}_{\mathrm{sym}}^{3 \times 3}$. Then the strain 
energy of the bulk body is given by $\left(\mu_{b}-\right.$ bulk shear modulus)

$$
E_{b}[\mathbf{u}]=\int_{\Omega_{0}} \mu_{b}\left|\frac{1}{2}\left[\nabla \mathbf{u}+(\nabla \mathbf{u})^{T}\right]-\boldsymbol{\epsilon}_{0}\right|^{2} d v
$$

where $\nabla \cdot \mathbf{u}=0$ in $\Omega_{0}$ due to incompressibility. From (1), we infer the following elastic stress-strain relation:

$$
\boldsymbol{\sigma}^{\mathrm{el}}=-p^{\mathrm{el}} \mathbf{I}+2 \mu_{b}\left[\frac{1}{2}\left[\nabla \mathbf{u}+(\nabla \mathbf{u})^{T}\right]-\boldsymbol{\epsilon}_{0}\right] \quad \text { in } \Omega_{0},
$$

where $p^{\text {el }}$ is the pressure or Lagrange's multiplier associated with the constraint $\nabla \cdot \mathbf{u}=0$.

Interior bulk dissipation is characterized by an incompressible viscous flow with the rate of energy dissipation given by ( $\eta^{\text {in }}-$ interior bulk viscosity)

$$
D^{\text {in }}[\mathbf{u}]=\int_{\Omega_{0}} 2 \eta^{\text {in }}\left|\frac{1}{2}\left[\nabla \dot{\mathbf{u}}+(\nabla \dot{\mathbf{u}})^{T}\right]\right|^{2} d v
$$

In other words, we have a local viscous stress-strain rate relation: ${ }^{1}$

$$
\boldsymbol{\sigma}^{\mathrm{vc}}=-p^{\mathrm{vc}} \mathbf{I}+\eta^{\mathrm{in}}\left[\nabla \dot{\mathbf{u}}+(\nabla \dot{\mathbf{u}})^{T}\right], \quad \nabla \cdot \dot{\mathbf{u}}=0 \quad \text { in } \Omega_{0} .
$$

All together, by (2) and (3) we write the interior stress-strain and strain rate relation as

$$
\boldsymbol{\sigma}^{\text {in }}=-p^{\text {in }} \mathbf{I}+\mu_{b}\left[\nabla \mathbf{u}+(\nabla \mathbf{u})^{T}-2 \boldsymbol{\epsilon}_{0}\right]+\eta^{\text {in }}\left[\nabla \dot{\mathbf{u}}+(\nabla \dot{\mathbf{u}})^{T}\right] \quad \text { in } \Omega_{0},
$$

where $p^{\mathrm{in}}=p^{\mathrm{el}}+p^{\mathrm{vc}}$ is the total interior pressure, and $\nabla \cdot \mathbf{u}=\nabla \cdot \dot{\mathbf{u}}=0$ due to incompressibility.

Exterior bulk dissipation is modelled as an incompressible viscous fluid with the rate of energy dissipation given by ( $\eta^{\text {ex }}-$ exterior bulk viscosity)

$$
D^{\mathrm{ex}}[\mathbf{u}]=\int_{\bar{\Omega}_{0}^{C}} 2 \eta^{\mathrm{ex}}\left|\frac{1}{2}\left[\nabla \dot{\mathbf{u}}+(\nabla \dot{\mathbf{u}})^{T}\right]\right|^{2} d v
$$

where $\bar{\Omega}_{0}^{C}=\mathbb{R}^{3} \backslash \bar{\Omega}_{0}$ is the exterior domain. In other words, we have a local stress-strain rate relation

$$
\boldsymbol{\sigma}^{\mathrm{ex}}=-p^{\mathrm{ex}} \mathbf{I}+\eta^{\mathrm{ex}}\left(\nabla \dot{\mathbf{u}}+(\nabla \dot{\mathbf{u}})^{T}\right), \quad \nabla \cdot \dot{\mathbf{u}}=0 \quad \text { in } \bar{\Omega}_{0}^{C},
$$

where $p^{\text {ex }}$ is the exterior pressure.

\subsection{Surface viscoelasticity}

To describe the properties of the membrane, we introduce a few notations. On the reference surface $\partial \Omega_{0}$, let $\boldsymbol{\nu}$ be the unit outward normal, $\mathbf{I}_{\nu}=\mathbf{I}-\boldsymbol{\nu} \otimes \boldsymbol{\nu}$ be the identity

\footnotetext{
${ }^{1}$ Strictly speaking, the exterior or interior fluids are not linear Newtonian fluids since we are employing Lagrangian coordinates.
} 
tensor on the tangent plane $\mathcal{T}_{\nu}:=\left\{\mathbf{e} \in \mathbb{R}^{3}: \mathbf{e} \cdot \boldsymbol{\nu}=0\right\}, \mathbb{S}_{\nu}=\left\{\mathbf{M} \in \mathbb{R}^{3 \times 3}: \mathbf{M} \boldsymbol{\nu}=\mathbf{M}^{T} \boldsymbol{\nu}=0\right\}$ be a $\boldsymbol{\nu}$-dependent subspace of $\mathbb{R}^{3 \times 3}$, and $\mathbb{P}_{\nu}: \mathbb{R}^{3 \times 3} \rightarrow \mathbb{S}_{\nu}$ be the orthogonal projection. Kinematically, the displacement $\mathbf{u}$ shall be continuously differentiable up to the boundary $\partial \Omega_{0}$, and we denote by

$$
u_{\nu}=\mathbf{u} \cdot \boldsymbol{\nu} \quad \text { and } \quad \mathbf{u}_{s}=\mathbf{u}-u_{\nu} \boldsymbol{\nu} \quad \text { on } \partial \Omega_{0}
$$

the normal and tangential components of the displacement $\mathbf{u}$ restricted to the boundary surface $\partial \Omega_{0}$, respectively. Also, it will be convenient to introduce surface gradient and divergence operators: ${ }^{2}$

$$
\nabla_{s}=\nabla-\boldsymbol{\nu}(\boldsymbol{\nu} \cdot \nabla) \quad \text { and } \quad \operatorname{div}_{s}=\operatorname{div}-\langle\boldsymbol{\nu}, \boldsymbol{\nu} \cdot \nabla\rangle .
$$

Then from the differential geometry we have the following identities: ${ }^{3}$

$$
\left\{\begin{array}{l}
\nabla_{s} \mathbf{u}=\nabla_{s} \mathbf{u}_{s}+\boldsymbol{\nu} \otimes \nabla_{s} u_{\nu}+u_{\nu} \mathbf{L} \\
\nabla_{s} \mathbf{u}_{s}=\mathbb{P}_{\nu}\left(\nabla_{s} \mathbf{u}_{s}\right)-\boldsymbol{\nu} \otimes\left(\mathbf{L u}_{s}\right) \\
\operatorname{div}_{s} \mathbf{u}=\operatorname{Tr}\left(\nabla_{s} \mathbf{u}\right)=\operatorname{div}_{s} \mathbf{u}_{s}+2 H u_{\nu}
\end{array}\right.
$$

where $\mathbf{L}=\nabla_{s} \boldsymbol{\nu}: \mathcal{T}_{\nu} \rightarrow \mathcal{T}_{\nu}$ is the Weingarten map (shape operator) on the tangential plane of the reference surface $\partial \Omega_{0}$, and $H=\frac{1}{2} \operatorname{Tr} \mathbf{L}$ is the mean curvature.

The membrane is modelled as a viscoelastic surface $\partial \Omega_{0}$ of zero-thickness coherently attached to the bulk body, meaning that there is no-slip between the interior and exterior materials and the membrane. Mathematically, these assumptions mean that both the displacement $\mathbf{u}$ and velocity $\dot{\mathbf{u}}$ are continuous up to the boundary $\partial \Omega_{0}$ from both the interior and the exterior side of $\partial \Omega_{0}$.

As for bulk elasticity, we postulate that the surface elastic (or strain) energy density $W_{s}$ depends only on the surface displacement gradient: $W_{s}=W_{s}\left(\nabla_{s} \mathbf{u}\right) .{ }^{4}$ Other interesting elastic models for fluid membranes will be discussed in Remarks 3. If the membrane is isotropic and the reference configuration is "close" to its natural surface-stress-free configuration, the (linearized) elastic energy of the membrane is necessarily given by (Gurtin and Murdoch, 1975; Hu and Liu, 2014)

$$
E_{s}[\mathbf{u}]=\int_{\partial \Omega_{0}} W_{s}\left(\nabla_{s} \mathbf{u}\right) d a, \quad W_{s}\left(\nabla_{s} \mathbf{u}\right)=W_{s}(0)+\boldsymbol{\sigma}_{s}^{0} \cdot \nabla_{s} \mathbf{u}+\frac{1}{2} \nabla_{s} \mathbf{u} \cdot \mathbb{C}_{s} \nabla_{s} \mathbf{u}
$$

where $\boldsymbol{\sigma}_{s}^{0}=\tau_{s}^{0} \mathbf{I}_{\nu} \in \mathbb{S}_{\nu}$ is the surface residual stress. Also, the surface stiffness tensor $\mathbb{C}_{s}$ shall satisfy (i) $\mathbb{P}_{\boldsymbol{\nu}} \mathbb{C}_{s} \mathbb{P}_{\boldsymbol{\nu}}=\mathbb{C}_{s}$, (ii) the major symmetry: $\mathbf{M}_{1} \cdot \mathbb{C}_{s} \mathbf{M}_{2}=\mathbf{M}_{2} \cdot \mathbb{C}_{s} \mathbf{M}_{1} \quad \forall \mathbf{M}_{1}, \mathbf{M}_{2} \in \mathbb{S}_{\nu}$,

\footnotetext{
${ }^{2}$ Note that the operators $\nabla_{s}$ and $\operatorname{div}_{s}$ are not covariant operators restricted to the tangent/cotangent space.

${ }^{3}$ Sign convention: the unit normal vector $\boldsymbol{\nu}$ is outward on $\partial \Omega_{0}, \mathbf{L}=\nabla_{s} \boldsymbol{\nu}$, and $H=\frac{1}{2} \operatorname{Tr} \mathbf{L}$, which is different from the usual convention in differential geometry, say, the textbook of Kühnel (2005). For a spherical surface of radius $R, H=1 / R$ and $\mathbf{L}=(\mathbf{I}-\boldsymbol{\nu} \otimes \boldsymbol{\nu}) / R=\mathbf{I}_{\nu} / R$.

${ }^{4}$ To include bending energy, we need to assume that $W_{s}$ depends on higher-order gradients, e.g., $\nabla_{s} \nabla_{s} \mathbf{u}$.
} 
and (iii) the minor symmetry:

$$
\mathbb{C}_{s} \mathbf{M}=0 \text { if } \mathbf{M}^{T}=-\mathbf{M}
$$

Moreover, if the surface is isotropic, then the surface stiffness tensor $\mathbb{C}_{s}$ shall be such that

$$
\frac{1}{2} \nabla_{s} \mathbf{u} \cdot \mathbb{C}_{s} \nabla_{s} \mathbf{u}=\frac{\mu_{s}}{4}\left|\mathbb{P}_{\nu}\left[\nabla_{s} \mathbf{u}+\left(\nabla_{s} \mathbf{u}\right)^{T}\right]\right|^{2}+\frac{\lambda_{s}}{2}\left|\operatorname{Tr}\left(\mathbb{P}_{\nu} \mathbf{u}\right)\right|^{2}
$$

where $\mu_{s}, \lambda_{s}$ are surface elastic moduli analogous to the Lamé constants in the bulk elasticity. In other words, the surface stress-strain relation is given by:

$$
\boldsymbol{\sigma}_{s}^{\mathrm{el}}=\boldsymbol{\sigma}_{s}^{0}+\mathbb{C}_{s} \nabla_{s} \mathbf{u}=\boldsymbol{\sigma}_{s}^{0}+\mu_{s} \mathbb{P}_{\nu}\left[\nabla_{s} \mathbf{u}+\left(\nabla_{s} \mathbf{u}\right)^{T}\right]+\lambda_{s}\left(\nabla_{s} \cdot \mathbf{u}\right) \mathbf{I}_{\nu}
$$

Similarly, we assume that the membrane is viscous with the rate of energy dissipation given by

$$
D_{s}[\mathbf{u}]=\int_{\partial \Omega_{0}} 2 \eta_{s}\left|\frac{1}{2} \mathbb{P}_{\nu}\left[\nabla_{s} \dot{\mathbf{u}}+\left(\nabla_{s} \dot{\mathbf{u}}\right)^{T}\right]\right|^{2}+\chi_{s}\left|\nabla_{s} \cdot \dot{\mathbf{u}}\right|^{2} d a
$$

where $\eta_{s}$ and $\chi_{s}$ are viscosities. In other words, the surface viscous stress-strain rate relation is given by

$$
\boldsymbol{\sigma}_{s}^{\mathrm{vc}}=\eta_{s} \mathbb{P}_{\nu}\left[\nabla_{s} \dot{\mathbf{u}}+\left(\nabla_{s} \dot{\mathbf{u}}\right)^{T}\right]+\chi_{s} \mathbf{I}_{\nu} \operatorname{div}_{s} \dot{\mathbf{u}}
$$

where $\eta_{s}, \chi_{s}$ are the associated surface viscosities of the membrane. Together with the elastic contribution, by (9) we write the surface stress-strain and strain rate relation as

$$
\begin{aligned}
\boldsymbol{\sigma}_{s}=\boldsymbol{\sigma}_{s}^{\mathrm{el}}+\boldsymbol{\sigma}_{s}^{\mathrm{vc}}=\boldsymbol{\sigma}_{s}^{0} & +\mu_{s} \mathbb{P}_{\nu}\left[\nabla_{s} \mathbf{u}+\left(\nabla_{s} \mathbf{u}\right)^{T}\right]+\lambda_{s} \mathbf{I}_{\nu} \operatorname{div}_{s} \mathbf{u} \\
& +\eta_{s} \mathbb{P}_{\nu}\left[\nabla_{s} \dot{\mathbf{u}}+\left(\nabla_{s} \dot{\mathbf{u}}\right)^{T}\right]+\chi_{s} \mathbf{I}_{\nu} \operatorname{div}_{s} \dot{\mathbf{u}} \quad \text { on } \partial \Omega_{0} .
\end{aligned}
$$

In general, material properties $\eta^{\text {in }}, \mu_{b}, \mu_{s}, \lambda_{s}, \eta_{s}, \chi_{s}, \eta^{\text {ex }}$ of heterogeneous bulk materials or surface membranes are position-dependent. For simplicity, we subsequently assume that they are position-independent, i.e., the interior, exterior and surface materials are respectively homogeneous.

Remark 1 (Pre-existing displacement). Because of the presence of residual stresses in the bulk and surface, the natural configuration of the body, i.e., the equilibrium configuration at the absence of any external loading, in general admits some nontrivial displacement $\mathbf{u}_{0}$ : $\Omega_{0} \rightarrow \mathbb{R}^{3}$ with reference to the configuration $\Omega_{0}$. This pre-existing displacement $\mathbf{u}_{0}$ can be determined by minimizing the free energy of the system:

$$
\min \left\{E_{b}[\mathbf{u}]+E_{s}[\mathbf{u}]: \int_{\Omega_{0}}|\nabla \mathbf{u}|^{2}+\int_{\partial \Omega_{0}}\left|\nabla_{s} \mathbf{u}\right|^{2}<+\infty\right\}
$$

By the standard first-variation calculation or the balance laws, we find that the pre-existing 
displacement $\mathbf{u}_{0}: \Omega_{0} \rightarrow \mathbb{R}^{3}$ shall satisfy the equilibrium equations:

$$
\begin{cases}\mu_{b} \Delta \mathbf{u}_{0}-\mu_{b} \operatorname{div} \boldsymbol{\epsilon}_{0}-\nabla p_{b}^{\text {in }}=0 & \text { in } \Omega_{0} \\ \operatorname{div}_{s} \boldsymbol{\sigma}_{s}=-\left(p^{\text {in }}-p^{\mathrm{ex}}\right) \boldsymbol{\nu}+\frac{1}{2} \mu_{b}\left[\nabla \mathbf{u}_{0}+\left(\nabla \mathbf{u}_{0}\right)^{T}-2 \boldsymbol{\epsilon}_{0}\right] \boldsymbol{\nu} & \text { on } \partial \Omega_{0}\end{cases}
$$

where the surface elastic stress $\boldsymbol{\sigma}_{s}=\boldsymbol{\sigma}_{s}^{0}+\mu_{s} \mathbb{P}_{\nu}\left[\nabla_{s} \mathbf{u}_{0}+\left(\nabla_{s} \mathbf{u}_{0}\right)^{T}\right]+\lambda_{s} \mathbf{I}_{\nu} \operatorname{div}_{s} \mathbf{u}_{0}$.

Remark 2 (Area conservation). If the surface area is locally conserved, we shall enforce the kinematic constraints:

$$
\nabla_{s} \cdot \mathbf{u}=\nabla_{s} \cdot \mathbf{u}_{s}+u_{\nu} 2 H=0 \quad \text { and } \quad \nabla_{s} \cdot \dot{\mathbf{u}}=\nabla_{s} \cdot \dot{\mathbf{u}}_{s}+\dot{u}_{\nu} 2 H=0 \quad \text { on } \partial \Omega_{0} .
$$

The surface elastic stress-strain relation can be written as

$$
\boldsymbol{\sigma}_{s}^{\mathrm{el}}=-p_{s}^{\mathrm{el}} \mathbf{I}_{\nu}+\boldsymbol{\sigma}_{s}^{0}+\mu_{s} \mathbb{P}_{\nu}\left[\nabla \mathbf{u}+(\nabla \mathbf{u})^{T}\right]
$$

whereas the surface viscous stress-strain rate relation reads

$$
\boldsymbol{\sigma}_{s}^{\mathrm{vc}}=-p_{s}^{\mathrm{vc}} \mathbf{I}_{\nu}+\eta_{s} \mathbb{P}_{\nu}\left[\nabla \dot{\mathbf{u}}+(\nabla \dot{\mathbf{u}})^{T}\right]
$$

All together, the surface stress is given by

$$
\boldsymbol{\sigma}_{s}=-p_{s} \mathbf{I}_{\nu}+\mu_{s} \mathbb{P}_{\nu}\left[\nabla \mathbf{u}+(\nabla \mathbf{u})^{T}\right]+\eta_{s} \mathbb{P}_{\nu}\left[\nabla \dot{\mathbf{u}}+(\nabla \dot{\mathbf{u}})^{T}\right] \quad \text { on } \partial \Omega_{0},
$$

where $p_{s}=p_{s}^{\mathrm{el}}+p_{s}^{\mathrm{vc}}-\tau_{s}^{0}$ can be interpreted as the in-membrane hydrostatic pressure.

Remark 3 (Lagrangian description vs Eulerian description). We employ the Lagrangian description in this work for coupling surface viscoelasticity with bulk viscoelasticity, though it would be more natural to use the Eulerian description for the exterior flow and fluid membrane (Seifert, 1997). Another advantage of the Lagrangian description lies in that the domain for our boundary value problems is fixed and independent of deformation.

The constitutive behavior of fluid membranes is commonly specified in the Eulerian description. For example, the surface tension model assumes that the elastic energy of the membrane is given by

$$
E_{s}[\partial \Omega]=\int_{\partial \Omega} \gamma d s
$$

where the constant $\gamma$ is referred to as the surface tension, and $\partial \Omega$ is the current configuration of the membrane. Another frequently used membrane model is the Helfrich-Canham bending model (Helfrich, 1973; Canham, 1970) with elastic energy given by

$$
E_{s}[\partial \Omega]=\int_{\partial \Omega}\left[2 \kappa_{b}\left(H-c_{*}\right)^{2}+\kappa_{g} G\right] d s
$$

where $c_{*}$ is the preferred mean curvature of the membrane, $H$ (resp. $G$ ) is the mean (resp. Gauss) curvature of the surface, and $\kappa_{b}$ (resp. $\left.\kappa_{g}\right)$ is the bending stiffness associated with the mean curvature (resp. Gauss) curvature. 
Remark 4 (Linearized surface tension model). To couple a fluid membrane with viscoelastic bulks, we need to translate the Eulerian model (18) or (19) to a Lagrangian model. The formal procedure is similar to the linearization of 3D elasticity and briefly outlined below. Let $\mathbf{y}: \Omega_{0} \rightarrow \Omega$ be the deformation (continuously differentiable up to the boundary), and $\mathbf{u}(\mathbf{x})=\mathbf{y}(\mathbf{x})-\mathbf{x}$ be the displacement. For the surface tension model (18), we consider a small strain in the sense that $\left|\nabla_{s} \mathbf{u}\right| \sim \varepsilon \ll 1$ on $\partial \Omega_{0}$. Then the surface energy (18) can be written as

$$
E_{s}[\partial \Omega]=\int_{\partial \Omega_{0}} \gamma J d a, \quad J=\sqrt{\operatorname{det}\left(\mathbf{C}_{s}\right)}
$$

where the surface Cauchy-Green tensor $\mathbf{C}_{s}=\left(\nabla_{s} \mathbf{y}\right)^{T} \nabla_{s} \mathbf{y}: \mathcal{T}_{\nu} \times \mathcal{T}_{\nu} \rightarrow \mathbb{R}$ is such that for any $\mathbf{e}_{1}, \mathbf{e}_{2} \in \mathcal{T}_{\nu}$

$$
\mathbf{C}_{s}\left(\mathbf{e}_{1} \otimes \mathbf{e}_{2}\right)=\left\langle\left(\nabla_{s} \mathbf{y}\right) \mathbf{e}_{1},\left(\nabla_{s} \mathbf{y}\right) \mathbf{e}_{2}\right\rangle .
$$

Expanding the integrand in (20) up to $O\left(\varepsilon^{2}\right)$, we obtain

$$
\begin{aligned}
\gamma J & =\gamma+\gamma \nabla_{s} \cdot \mathbf{u}+\frac{\gamma}{2}\left[\left|\nabla_{s} \mathbf{u}\right|^{2}+\operatorname{det}\left\{\mathbb{P}_{\nu}\left[\nabla_{s} \mathbf{u}+\left(\nabla_{s} \mathbf{u}\right)^{T}\right]\right\}-\left(\nabla_{s} \cdot \mathbf{u}\right)^{2}\right]+o\left(\varepsilon^{2}\right) \\
& =\gamma+\gamma \mathbf{I}_{\nu} \cdot \nabla_{s} \mathbf{u}+\frac{\gamma}{2}\left[2 \operatorname{det}\left(\mathbb{P}_{\nu} \nabla_{s} \mathbf{u}\right)+\left|\nabla_{s} \mathbf{u}\right|^{2}-\left|\mathbb{P}_{\nu} \nabla_{s} \mathbf{u}\right|^{2}\right]+o\left(\varepsilon^{2}\right) \\
& =\gamma+\gamma \mathbf{I}_{\nu} \cdot \nabla_{s} \mathbf{u}+\frac{1}{2} \nabla_{s} \mathbf{u} \cdot \mathbb{C}_{s} \nabla_{s} \mathbf{u}+o\left(\varepsilon^{2}\right),
\end{aligned}
$$

from which the "surface residual stress" and (resp. "surface stiffness tensor") is identified as ${ }^{5}$

$$
\begin{aligned}
& \boldsymbol{\sigma}_{s}^{0}=\gamma \mathbf{I}_{\nu} \quad \text { and } \quad(\text { resp } . \\
& \left.\mathbb{C}_{s} \nabla_{s} \mathbf{u}=\gamma\left[\nabla_{s} \mathbf{u}+\left(\nabla_{s} \cdot \mathbf{u}\right) \mathbf{I}_{\nu}-\mathbb{P}_{\nu} \nabla_{s} \mathbf{u}-\left(\mathbb{P}_{\nu} \nabla_{s} \mathbf{u}\right)^{T}\right]\right) .
\end{aligned}
$$

We note that the natural energy-minimizing state associated with (20) is a surface of zero area and not close to the reference configuration $\partial \Omega_{0}$, and henceforth, the surface elasticity tensor in $(21)_{2}$ does not have to satisfy the minor symmetry (8). For small strains, it is clear that the second term in (21), i.e., the surface residual stress $\gamma \mathbf{I}_{\nu}$, dominates the third term which is quadratic in surface strain $\nabla_{s} \mathbf{u}$. There is, however, an important exception when the interior material is incompressible and the reference configuration $\Omega_{0}$ is a sphere.

Remark 5. If the encapsulated volume of the membrane $\operatorname{vol}(\Omega)$ is conserved, we have

$$
\int_{\Omega_{0}} \operatorname{det}(\nabla \mathbf{y}) d v=\int_{\Omega_{0}}\left(1+\nabla \cdot \mathbf{u}+\frac{1}{2} \nabla \mathbf{u} \cdot \mathbb{T} \nabla \mathbf{u}\right) d v+o\left(\varepsilon^{2}\right)=\int_{\Omega_{0}} 1 d v
$$

where $\mathbb{T}: \mathbb{R}^{3 \times 3} \rightarrow \mathbb{R}^{3 \times 3}$ is a fourth-order symmetric tensor such that $\frac{1}{2} \mathbf{F} \cdot \mathbb{T} \mathbf{F}=\sum_{i<j} \sum_{j=1}^{3} \lambda_{i} \lambda_{j}$

\footnotetext{
${ }^{5}$ From the viewpoint of the Lagrangian description, the terminology "surface tension" is particularly overloaded in the sense that the surface energy per unit area of the undeformed surface, residual surface stress, and surface stiffness tensor/modulus are all proportional to the surface tension $\gamma$.
} 
is the second invariant of matrix $\mathbf{F}\left(\lambda_{i}\right.$ are the eigenvalues of $\mathbf{F}$ ), i.e.,

$$
\mathbb{T} \mathbf{F}=(\operatorname{Tr} \mathbf{F}) \mathbf{I}-\mathbf{F}^{T} \quad \forall \mathbf{F} \in \mathbb{R}^{3 \times 3} .
$$

By the divergence theorem, equation (23) implies that

$$
\begin{aligned}
o\left(\varepsilon^{2}\right) & =\int_{\Omega_{0}}\left(\nabla \cdot \mathbf{u}+\frac{1}{2} \nabla \mathbf{u} \cdot \mathbb{T} \nabla \mathbf{u}\right) d a=\int_{\partial \Omega_{0}}\left[u_{\nu}+\frac{1}{2} \mathbf{u} \cdot(\mathbb{T} \nabla \mathbf{u}) \boldsymbol{\nu}\right] d a \\
& =\int_{\partial \Omega_{0}}\left\{u_{\nu}+\frac{1}{2}\left[u_{\nu} \nabla_{s} \cdot \mathbf{u}-\boldsymbol{\nu} \cdot\left(\nabla_{s} \mathbf{u}\right) \mathbf{u}_{s}\right]\right\} d a
\end{aligned}
$$

where the last equality follows from (24):

$$
\mathbf{u} \cdot(\mathbb{T} \nabla \mathbf{u}) \boldsymbol{\nu}=u_{\nu} \nabla \cdot \mathbf{u}-\boldsymbol{\nu} \cdot(\nabla \mathbf{u})\left(u_{\nu} \boldsymbol{\nu}+\mathbf{u}_{s}\right)=u_{\nu} \nabla_{s} \cdot \mathbf{u}-\boldsymbol{\nu} \cdot\left(\nabla_{s} \mathbf{u}\right) \mathbf{u}_{s}
$$

From (25), we see that $\int_{\partial \Omega_{0}} u_{\nu} d a \sim O\left(\varepsilon^{2}\right)$ because of the volume constraint (23).

Moreover, from (21) the elastic energy contributed by the surface residual stress is given by

$$
\int_{\partial \Omega_{0}} \boldsymbol{\sigma}_{s}^{0} \cdot \nabla_{s} \mathbf{u} d a=\int_{\partial \Omega_{0}} \gamma \operatorname{div}_{s} \mathbf{u} d a=\int_{\partial \Omega_{0}} \gamma\left(\operatorname{div}_{s} \mathbf{u}_{s}+\boldsymbol{\nu} \cdot \mathbf{u} H\right) d a=\gamma \int_{\partial \Omega_{0}} u_{\nu} H d a .
$$

If the reference surface $\partial \Omega_{0}$ is a spherical surface, the mean curvature $H=$ const. on $\partial \Omega_{0}$ and hence the integral (26) is of the same order as the third term in (21). Therefore, upon eliminating $\int_{\partial \Omega_{0}} \nabla_{s} \cdot \mathbf{u} d a=\int_{\partial \Omega_{0}} 2 H u_{\nu} d a$ by (25) and neglecting higher-order terms $o\left(\varepsilon^{2}\right)$, we obtain the linearized elastic energy for constant-volume quasi-spherical membranes associated with constant surface tension model:

$$
\begin{aligned}
E_{s}[\mathbf{u}] & =\gamma \int_{\partial \Omega_{0}} \sqrt{\operatorname{det}(\mathbf{C})} d a \\
& =\frac{\gamma}{2} \int_{\partial \Omega_{0}}\left[2 \operatorname{det}\left(\mathbb{P}_{\nu} \nabla_{s} \mathbf{u}\right)+\left|\nabla_{s} \mathbf{u}\right|^{2}-\left|\mathbb{P}_{\nu} \nabla_{s} \mathbf{u}\right|^{2}-2 H u_{\nu} \nabla_{s} \cdot \mathbf{u}+2 H \boldsymbol{\nu} \cdot\left(\nabla_{s} \mathbf{u}\right) \mathbf{u}_{s}\right] d a
\end{aligned}
$$

where an additive constant independent of the displacement and terms beyond $O\left(\varepsilon^{2}\right)$ have been neglected in the last equality.

Remark 6 (Linearized Helfrich model). We now consider the Helfrich model (19). Restricting ourselves to quasi-spherical closed surfaces, the contribution from Gauss curvature is constant and can be omitted. For simplicity, we choose the spherical surface $\partial \Omega_{0}$ of radius $R=1 / c_{0}$ as the reference configuration and assume $c_{0}^{-1}\left(c_{0}-c_{*}\right) \sim \varepsilon \ll 1$. Consider a deformation $\mathbf{y}: \partial \Omega_{0} \rightarrow \partial \Omega$. Then the Helfrich energy (19) can be written as ${ }^{6}$

$$
E_{s}[\mathbf{y}]=\int_{\partial \Omega_{0}} 2 \kappa_{b}\left(\tilde{H}-c_{*}\right)^{2} J d a
$$

\footnotetext{
${ }^{6}$ Throughout this paper, we assume that the membrane surface has no topological changes, and hence by the Gauss-Bonnet theorem, $\int_{\partial \Omega} G d s$ is constant.
} 
where $\left(\tilde{\boldsymbol{\nu}}=\mathbf{y}_{, 1} \times \mathbf{y}_{, 2} /\left|\mathbf{y}_{, 1} \times \mathbf{y}_{, 2}\right|\right.$ is the unit normal on $\left.\partial \Omega\right)$

$$
\tilde{\mathbf{L}}=-\left\langle\tilde{\boldsymbol{\nu}}, \nabla_{s} \nabla_{s} \mathbf{y}\right\rangle, \quad 2 \tilde{H}=\operatorname{Tr}(\tilde{\mathbf{L}}) .
$$

If the strain is small in the sense that $\mathbf{u}(\mathbf{x})=\mathbf{y}(\mathbf{x})-\mathbf{x}$ and $\left|\nabla_{s} \mathbf{u}\right| \sim \varepsilon \ll 1$, then for the displaced surface parametrized by $\mathbf{y}(\mathbf{x})=\mathbf{x}+\mathbf{u}(\mathbf{x}), \mathbf{x} \in \partial \Omega_{0}$, we have that (Steigmann et al., 2003):

$$
c_{0}^{-1} \tilde{H}=1+c_{0}^{-1}\left(\frac{1}{2} \Delta_{s} u_{\nu}+c_{0}^{2} u_{\nu}\right)+o(\varepsilon), \quad J=1+c_{0} \nabla_{s} \cdot \mathbf{u}+o(\varepsilon) .
$$

Therefore, the linearized Helfrich energy in terms of displacement is given by

$$
E_{s}[\mathbf{u}]=\frac{\kappa_{b}}{2} \int_{\partial \Omega_{0}}\left(\Delta_{s} u_{\nu}+2 c_{0}^{2} u_{\nu}\right)^{2} d a,
$$

where we have neglected an additive constant independent of the displacement and terms beyond $O\left(\varepsilon^{2}\right)$. In particular, we have noticed that for the spherical reference surface $\partial \Omega_{0}$,

$$
\int_{\partial \Omega_{0}}\left(c_{0}-c_{*}\right)\left(\frac{1}{2} \Delta_{s} u_{\nu}+c_{0}^{2} u_{\nu}\right) d a=o\left(\varepsilon^{2}\right) .
$$

\section{Balance laws}

\subsection{Balance of linear momentum}

We first consider general time-dependent processes. For the moment, we consider only two kinds of external loadings: a external body force $\mathbf{b}: \Omega_{0} \rightarrow \mathbb{R}^{3}$ and a surface traction $\mathbf{t}_{s}: \partial \Omega_{0} \rightarrow \mathbb{R}$. More general loading conditions will be discussed in Section 4 .

Neglecting the effects of inertia and gravity, from the balance of linear momentum and (4) we have that in the interior domain,

$$
\operatorname{div} \boldsymbol{\sigma}^{\text {in }}=\mu_{b} \Delta \mathbf{u}+\eta^{\text {in }} \Delta \dot{\mathbf{u}}-2 \mu_{b} \operatorname{div} \boldsymbol{\epsilon}_{0}-\nabla p^{\text {in }}=-\mathbf{b} \quad \text { in } \Omega_{0} .
$$

For the exterior domain, by (5) we have

$$
\operatorname{div} \boldsymbol{\sigma}^{\mathrm{ex}}=\eta^{\mathrm{ex}} \Delta \dot{\mathbf{u}}-\nabla p^{\mathrm{ex}}=0 \quad \text { in } \bar{\Omega}_{0}^{C} .
$$

For the membrane $\partial \Omega_{0}$, we have the generalized Young-Laplace equation:

$$
\operatorname{div}_{s}\left(\boldsymbol{\sigma}_{s}\right)=\left(\boldsymbol{\sigma}^{\mathrm{in}}-\boldsymbol{\sigma}^{\mathrm{ex}}\right) \boldsymbol{\nu}-\mathbf{t}_{s} \quad \text { on } \partial \Omega_{0} .
$$

As discussed in Remark 1 , the body in general admits a nontrivial initial displacement $\mathbf{u}_{0}(\mathbf{x})$ with respect to the reference configuration $\Omega_{0}$. In account of the constitutive relations (4), (5) and (12) (or (17)) and upon specifying suitable boundary conditions and initial conditions, e.g.,

$$
p^{\mathrm{ex}}, \mathbf{u}, \dot{\mathbf{u}} \rightarrow 0 \quad \text { as } \quad|\mathbf{x}| \rightarrow+\infty \quad \text { and } \quad \mathbf{u}(\mathbf{x}, t=0)=\mathbf{u}_{0}(\mathbf{x})
$$


we shall be able to solve (30), (31) and (32) for the displacement $\mathbf{u}(\mathbf{x}, t)$, exterior pressure $p^{\mathrm{ex}}(\mathbf{x}, t)$ and interior pressure $p^{\text {in }}(\mathbf{x}, t)$.

Remark 7. We notice that (30)-(33), together with the constitutive relations (4), (5) and (12) (or (17)), form a linear boundary value problem for $\mathbf{u}(\mathbf{x}, t)$. Therefore, the displacement $\mathbf{w}(\mathbf{x}, t):=\mathbf{u}(\mathbf{x}, t)-\mathbf{u}_{0}(\mathbf{x})$ relative to the body's natural but deformed configuration is independent of the eigenstrain $\boldsymbol{\epsilon}_{0}$ and surface residual stress $\boldsymbol{\sigma}_{s}^{0}$. Experimentally, we can only measure the relative displacement $\mathbf{w}(\mathbf{x}, t)$ which is precisely determined by (30)-(33) with vanishing eigenstrain and surface residual stress (i.e., $\boldsymbol{\epsilon}_{0}=0$ and $\boldsymbol{\sigma}_{s}^{0}=0$ ). For brevity and without loss of generality, we subsequently set $\boldsymbol{\epsilon}_{0}=0, \boldsymbol{\sigma}_{s}^{0}=0$ and the initial displacement $\mathbf{u}_{0}=0$.

\subsection{Balance of energy}

Let $\mathcal{P} \subset \Omega_{0}$ be an arbitrary subdomain. By the divergence theorem and (30) we find that

$$
\begin{aligned}
\frac{d}{d t} \int_{\mathcal{P}} \mu_{b}\left|\frac{1}{2}\left[\nabla \mathbf{u}+(\nabla \mathbf{u})^{T}\right]\right|^{2} d v & =\int_{\mathcal{P}} \boldsymbol{\sigma}_{b}^{\mathrm{el}} \cdot \nabla \dot{\mathbf{u}} d v \\
& =\int_{\partial \mathcal{P}} \boldsymbol{\nu} \cdot \boldsymbol{\sigma}_{b}^{\mathrm{el}} \dot{\mathbf{u}} d a-\int_{\mathcal{P}} \dot{\mathbf{u}} \cdot \operatorname{div} \boldsymbol{\sigma}_{b}^{\mathrm{el}} d v \\
& =\int_{\partial \mathcal{P}} \boldsymbol{\nu} \cdot \boldsymbol{\sigma}_{b}^{\mathrm{el}} \dot{\mathbf{u}} d a+\int_{\mathcal{P}} \dot{\mathbf{u}} \cdot\left(\operatorname{div} \boldsymbol{\sigma}_{b}^{\mathrm{vc}}+\mathbf{b}-\nabla p\right) d v \\
& =-2 \eta^{\mathrm{in}} \int_{\mathcal{P}}|\nabla \dot{\mathbf{u}}|^{2} d v+\int_{\partial \mathcal{P}} \dot{\mathbf{u}} \cdot \boldsymbol{\sigma}^{\mathrm{in}} \boldsymbol{\nu} d a+\int_{\mathcal{P}} \dot{\mathbf{u}} \cdot \mathbf{b} d v
\end{aligned}
$$

where the left hand side can be recognized as the rate of change of the bulk strain energy in $\mathcal{P}$, the first term on the right hand side can be identified as the rate of energy dissipation due to viscosity, and the second and third term can be identified as the rate of work done to $\mathcal{P}$ by the exterior of $\mathcal{P}$ and by the external body force $\mathbf{b}$, respectively. Similarly, for any exterior subdomain $\mathcal{P} \subset\left(\mathbb{R}^{3} \backslash \Omega_{0}\right)$, we have

$$
0=-2 \eta^{\mathrm{ex}} \int_{\mathcal{P}}|\nabla \dot{\mathbf{u}}|^{2} d v+\int_{\partial \mathcal{P}} \dot{\mathbf{u}} \cdot \boldsymbol{\sigma}^{\mathrm{ex}} \boldsymbol{\nu} d a .
$$

For the surface $\partial \Omega_{0}$, by (30), (31), (32), and (12) we have

$$
\begin{aligned}
\int_{\partial \Omega_{0}} \dot{\mathbf{u}} \cdot\left[\left(\boldsymbol{\sigma}^{\mathrm{in}}-\boldsymbol{\sigma}^{\mathrm{ex}}\right) \boldsymbol{\nu}-\mathbf{t}_{s}\right] d a & =\int_{\partial \Omega_{0}} \dot{\mathbf{u}} \cdot \operatorname{div}_{s} \boldsymbol{\sigma}_{s} d a=\int_{\partial \Omega_{0}}\left[\operatorname{div}_{s}\left(\boldsymbol{\sigma}_{s} \dot{\mathbf{u}}\right)-\boldsymbol{\sigma}_{s} \cdot \nabla_{s} \dot{\mathbf{u}}\right] d a \\
& =-\int_{\partial \Omega_{0}}\left(\boldsymbol{\sigma}_{s}^{\mathrm{el}}+\boldsymbol{\sigma}_{s}^{\mathrm{vc}}\right) \cdot \nabla_{s} \dot{\mathbf{u}} d a \\
& =-\frac{d}{d t} \int_{\partial \Omega_{0}} \frac{1}{2} \nabla_{s} \mathbf{u} \cdot \mathbb{C}_{s} \nabla_{s} \mathbf{u} d a-D_{s}[\mathbf{u}] \\
& =-\frac{d}{d t} E_{s}[\mathbf{u}]-D_{s}[\mathbf{u}]
\end{aligned}
$$


All together, by (34)-(36) we have the expected energy balance equation for the entire system:

$$
\begin{aligned}
\frac{d}{d t}\left(E_{b}[\mathbf{u}]+E_{s}[\mathbf{u}]\right) & =-2 \eta^{\text {in }} \int_{\Omega_{0}}|\nabla \dot{\mathbf{u}}|^{2} d v-2 \eta^{\operatorname{ex}} \int_{\bar{\Omega}_{0}^{C}}|\nabla \dot{\mathbf{u}}|^{2} d v-D_{s}[\mathbf{u}]+Q[\mathbf{u}] \\
& =-D^{\text {in }}[\mathbf{u}]-D^{\operatorname{ex}}[\mathbf{u}]-D_{s}[\mathbf{u}]+Q[\mathbf{u}]
\end{aligned}
$$

where $Q[\mathbf{u}]$ denotes the rate of work done by external forces:

$$
Q[\mathbf{u}]=\int_{\Omega_{0}} \mathbf{b} \cdot \dot{\mathbf{u}} d v+\int_{\partial \Omega_{0}} \mathbf{t}_{s} \cdot \dot{\mathbf{u}} d a .
$$

\section{Explicit solutions for quasi-spherical vesicles}

\subsection{General solutions}

We now present explicit solutions to (30), (31) and (32) for quasi-spherical bodies at the absence of body force $\mathbf{b}=0$. We first outline the general solution procedure. Setting $\tilde{\mathbf{u}}=\mathbf{u}+\tau_{0} \dot{\mathbf{u}}\left(\tau_{0}=\eta^{\text {in }} / \mu_{b}\right)$ we rewrite the interior equation (30) as

$$
\nabla \cdot \tilde{\mathbf{u}}=0, \quad \mu_{b} \Delta \tilde{\mathbf{u}}-\nabla p^{\text {in }}=0 \quad \text { in } \Omega_{0} .
$$

One may recognize the above equation as the usual Stokes flow inside $\Omega_{0}$ or the equilibrium equation for an incompressible solid. For a given boundary condition:

$$
\mathbf{u}(\mathbf{x}, t)=\mathbf{u}^{*}(\mathbf{x}, t) \quad \forall \mathbf{x} \in \partial \Omega_{0}, t \in[0,+\infty),
$$

we can solve (38) for $\tilde{\mathbf{u}}(\mathbf{x}, t)$ in $\Omega_{0}$. Further, we can determine the interior traction on the surface which defines a mapping $\left.\tilde{\mathbf{u}}\right|_{\partial \Omega_{0}} \mapsto \mu_{b} \operatorname{DN}^{\text {in }}\left(\left.\tilde{\mathbf{u}}\right|_{\partial \Omega_{0}}\right):=\left.\boldsymbol{\sigma}^{\text {in }} \boldsymbol{\nu}\right|_{\partial \Omega_{0}}:{ }^{7}$

$$
\mu_{b} \operatorname{DN}^{\text {in }}\left(\mathbf{u}^{*}+\tau_{0} \dot{\mathbf{u}}^{*}\right)=\left.\left\{\mu_{b}\left[\nabla \tilde{\mathbf{u}}+(\nabla \tilde{\mathbf{u}})^{T}\right] \boldsymbol{\nu}-p^{\text {in }} \boldsymbol{\nu}\right\}\right|_{\partial \Omega_{0}} .
$$

Similarly, for the exterior domain we have

$$
\nabla \cdot \dot{\mathbf{u}}=0, \quad \eta^{\mathrm{ex}} \Delta \dot{\mathbf{u}}-\nabla p^{\mathrm{ex}}=0 \quad \text { in } \mathbb{R}^{3} \backslash \bar{\Omega}_{0}
$$

and upon solving the above problem we determine the exterior traction on the membrane which defines a mapping $\left.\dot{\mathbf{u}}\right|_{\partial \Omega_{0}} \mapsto \eta^{\mathrm{ex}} \operatorname{DN}^{\mathrm{ex}}\left(\left.\dot{\mathbf{u}}\right|_{\partial \Omega_{0}}\right):=-\left.\boldsymbol{\sigma}^{\mathrm{ex}} \boldsymbol{\nu}\right|_{\partial \Omega_{0}}$ :

$$
\eta^{\mathrm{ex}} \mathrm{DN}^{\mathrm{ex}}\left(\dot{\mathbf{u}}^{*}\right)=\left.\left\{-\eta^{\mathrm{ex}}\left[\nabla \dot{\mathbf{u}}+(\nabla \dot{\mathbf{u}})^{T}\right] \boldsymbol{\nu}+p^{\mathrm{ex}} \boldsymbol{\nu}\right\}\right|_{\partial \Omega_{0}} .
$$

Inserting (41)-(40) into (32) we obtain a system of differential equations:

$$
\left\{\begin{array}{c}
\operatorname{div}_{s}\left(\boldsymbol{\sigma}_{s}\right)=\mu_{b} \mathrm{DN}^{\mathrm{in}}\left(\mathbf{u}^{*}+\tau_{0} \dot{\mathbf{u}}^{*}\right)+\eta^{\mathrm{ex}} \mathrm{DN}^{\mathrm{ex}}\left(\dot{\mathbf{u}}^{*}\right)-\mathbf{t}_{s} \\
\boldsymbol{\sigma}_{s}=\mu_{s} \mathbb{P}_{\nu}\left[\nabla_{s} \mathbf{u}+\left(\nabla_{s} \mathbf{u}\right)^{T}\right]+\lambda_{s} \mathbf{I}_{\nu} \operatorname{div}_{s} \mathbf{u} \\
+\eta_{s} \mathbb{P}_{\nu}\left[\nabla_{s} \dot{\mathbf{u}}+\left(\nabla_{s} \dot{\mathbf{u}}\right)^{T}\right]+\chi_{s} \mathbf{I}_{\nu} \operatorname{div}_{s} \dot{\mathbf{u}}
\end{array} \quad \forall(\mathbf{x}, t) \in \partial \Omega_{0} \times(0,+\infty)\right.
$$

\footnotetext{
${ }^{7}$ This is precisely the so-called Dirichlet-to-Neumann map.
} 
where we have employed the linearized Gurtin-Murdoch surface elasticity model (12). We remark that the system (42) for the displacement restricted to the surface $\partial \Omega_{0}$, i.e., $\mathbf{u}$ : $\partial \Omega_{0} \times[0,+\infty) \rightarrow \mathbb{R}$, is nonlocal but linear, and presumably admits a unique solution for reasonable initial conditions.

To achieve explicit solutions to (42), we consider quasi-spherical bodies and choose the reference configuration to be a ball of radius $R$ : $\Omega_{0}=B_{R}$. For the spherical surface $\partial B_{R}$, we have that

shape operator $\mathbf{L}=H \mathbf{I}_{\nu}, \quad$ mean curvature $H=1 / R, \quad$ and $\quad \operatorname{div}_{s} \mathbf{L}=-2 H^{2} \boldsymbol{\nu}$.

We will restrict ourselves to axis-symmetric scenarios and write the applied traction $\mathbf{t}_{s}(\cdot, t)$ : $\partial B_{R} \rightarrow \mathbb{R}^{3}$, the vector field $\mathbf{u}(\cdot, t): \mathbb{R}^{3} \rightarrow \mathbb{R}^{3}$ and the scalar pressure $p(\cdot, t): \mathbb{R}^{3} \rightarrow \mathbb{R}$ in terms of (vector) spherical harmonics as (see Appendix A):

$$
\begin{aligned}
& \mathbf{t}_{s}(\mathbf{x}, t)=\sum_{l=0}^{\infty}\left[t_{l}^{r}(t) \mathbf{Y}_{l}(\theta)+t_{l}^{\theta}(t) \mathbf{\Psi}_{l}(\theta)\right] \\
& \mathbf{u}(\mathbf{x}, t)=\sum_{l=0}^{\infty}\left[u_{l}^{r}(r, t) \mathbf{Y}_{l}(\theta)+u_{l}^{\theta}(r, t) \mathbf{\Psi}_{l}(\theta)\right], \quad p(\mathbf{x})=\sum_{l=0}^{\infty} p_{l}(r) Y_{l}(\theta)
\end{aligned}
$$

where $(r, \theta, \varphi)$ are the the usual spherical coordinates,

$$
Y_{l}(\theta)=\sqrt{\frac{2 l+1}{4 \pi}} P_{l}(\cos \theta), \quad P_{l}(x)=\frac{1}{2^{l} l !} \frac{d^{l}}{d x^{l}}\left[\left(x^{2}-1\right)^{l}\right],
$$

and the axis-symmetric vector spherical harmonics are defined as $\left(\hat{\mathbf{r}}=\mathbf{r} / r, \mathbf{e}_{\theta}, \mathbf{e}_{\varphi}\right.$ are the unit basis vectors associated with the spherical coordinates $(r, \theta, \varphi)$ )

$$
\mathbf{Y}_{l}=Y_{l} \hat{\mathbf{r}}, \quad \mathbf{\Psi}_{l}=r \nabla Y_{l}=Y_{l}^{\prime} \mathbf{e}_{\theta}, \quad \boldsymbol{\Phi}_{l}=\mathbf{r} \times \nabla Y_{l}=Y_{l}^{\prime} \mathbf{e}_{\varphi}
$$

For brevity, denote the boundary values of $\mathbf{u}(\mathbf{x}, t)$ on $\partial B_{R}$ by

$$
\left.\left[\begin{array}{l}
u_{l}^{r}(r, t) \\
u_{l}^{\theta}(r, t)
\end{array}\right]\right|_{r=R}=\left[\begin{array}{l}
X_{l}(t) \\
\Theta_{l}(t)
\end{array}\right]=: \phi_{l}(t), \quad \mathbf{f}_{l}=\left[\begin{array}{c}
t_{l}^{r}(t) \\
l(l+1) t_{l}^{\theta}(t)
\end{array}\right] .
$$

By Appendix A-B, (A.8) and (B.23), and pre-multiplying $(42)_{1}$ by $R^{2} \operatorname{diag}[1, l(l+1)]$, we obtain that $(l=0,1,2, \cdots)$

$$
\mathbf{D}_{l} \dot{\phi}_{l}+\mathbf{S}_{l} \phi_{l}=R^{2} \mathbf{f}_{l}
$$


where $2 \times 2$ symmetric matrices $\mathbf{D}_{l}, \mathbf{S}_{l}$ are defined as (c.f., $($ A.10), (B.25), $\tilde{l}=l(l+1))$

$$
\begin{aligned}
& \mathbf{D}_{l}=\eta^{\text {in }} R \mathbf{D}_{l}^{\text {in }}+\eta^{\text {ex }} R \mathbf{D}_{l}^{\text {ex }}+\eta_{s} \mathbf{S}_{l}^{a}+\chi_{s} \mathbf{S}_{l}^{b}, \\
& \mathbf{S}_{l}=2 \mu_{s} \mathbf{S}_{l}^{a}+\lambda_{s} \mathbf{S}_{l}^{b}+\mu_{b} R \mathbf{D}_{l}^{\text {in }}, \\
& \mathbf{D}_{l}^{\text {in }}=\left[\begin{array}{cc}
1+\frac{3}{l}+2 l & -3(1+l) \\
-3(l+1) & l(l+1)(2 l+1)
\end{array}\right], \quad \mathbf{D}_{l}^{\mathrm{ex}}=\left[\begin{array}{cc}
\frac{2 l^{2}+3 l+4}{l+1} & -3 l \\
-3 l & l(l+1)(2 l+1)
\end{array}\right], \\
& \mathbf{S}_{l}^{a}=\left[\begin{array}{cc}
2 & -\tilde{l} \\
-\tilde{l} & \tilde{l}^{2}-\tilde{l}
\end{array}\right], \quad \mathbf{S}_{l}^{b}=\left[\begin{array}{cc}
4 & -2 \tilde{l} \\
-2 \tilde{l} & \tilde{l}^{2}
\end{array}\right] .
\end{aligned}
$$

Let $\mathbf{K}_{l}=\mathbf{D}_{l}^{-1} \mathbf{S}_{l}$. By a Laplace transformation we find that the solution to (47) is explicitly given by

$$
\phi_{l}(t)=\exp \left(-t \mathbf{K}_{l}\right)\left[\boldsymbol{\phi}_{l}(0)+R^{2} \int_{0}^{t} \exp \left(\tau \mathbf{K}_{l}\right) \mathbf{D}_{l}^{-1} \mathbf{f}_{l}(\tau) d \tau\right]
$$

If the external surface traction is independent of time, then $\mathbf{f}_{l}$ is a constant vector and the above solution can be rewritten as

$$
\boldsymbol{\phi}_{l}(t)=\exp \left(-t \mathbf{K}_{l}\right) \boldsymbol{\phi}_{l}(0)+R^{2}\left[\mathbf{I}-\exp \left(-t \mathbf{K}_{l}\right)\right] \mathbf{S}_{l}^{-1} \mathbf{f}_{l},
$$

where $\mathbf{I} \in \mathbb{R}^{2 \times 2}$ is the identity matrix. Moreover, if the external surface traction is sinusoidal, i.e., $\mathbf{f}_{l}(t)=\mathbf{f}_{l}^{0} e^{i \omega t}$, by direct integration we find the solution (49) can be written as

$$
\boldsymbol{\phi}_{l}(t)=\exp \left(-t \mathbf{K}_{l}\right) \boldsymbol{\phi}_{l}(0)+R^{2}\left(e^{i \omega t} \mathbf{I}-e^{-t \mathbf{K}_{l}}\right)\left(\mathbf{S}_{l}+i \omega \mathbf{D}_{l}\right)^{-1} \mathbf{f}_{l}^{0} .
$$

Remark 8 (Energetics). It is worthwhile to mention that the momentum balance equation (47) is consistent with the energy balance equation (37). For the specified displacement and velocity $\mathbf{u}^{*}$ and $\dot{\mathbf{u}}^{*}$ on $\partial B_{R}$ as in (46), we find each of the terms in (37) as:

$$
\begin{aligned}
E_{s}[\mathbf{u}] & =\int_{\partial \Omega_{0}} \frac{1}{2} \nabla_{s} \mathbf{u} \cdot \mathbb{C}_{s} \nabla_{s} \mathbf{u} d a=\sum_{l=0}^{\infty}\left(\mu_{s} \boldsymbol{\phi}_{l} \cdot \mathbf{S}_{l}^{a} \boldsymbol{\phi}_{l}+\frac{\lambda_{s}}{2} \boldsymbol{\phi}_{l} \cdot \mathbf{S}_{l}^{b} \boldsymbol{\phi}_{l}\right) \\
E_{b}[\mathbf{u}] & =\frac{1}{2} \int_{\Omega_{0}} \mu_{b}\left|\nabla_{s} \mathbf{u}\right|^{2} d a=R \sum_{l=0}^{\infty} \frac{\mu_{b}}{2} \boldsymbol{\phi}_{l} \cdot \mathbf{D}_{l}^{\text {in }} \boldsymbol{\phi}_{l} \\
D_{s}[\mathbf{u}] & =\int_{\partial \Omega_{0}} 2 \eta_{s}\left|\frac{1}{2} \mathbb{P}_{\nu}\left[\nabla_{s} \dot{\mathbf{u}}+\left(\nabla_{s} \dot{\mathbf{u}}\right)^{T}\right]\right|^{2}+\chi_{s}\left|\nabla_{s} \cdot \dot{\mathbf{u}}\right|^{2} d a \\
& =\sum_{l=0}^{\infty}\left(2 \eta_{s} \dot{\boldsymbol{\phi}}_{l} \cdot \mathbf{S}_{l}^{a} \dot{\boldsymbol{\phi}}_{l}+\chi_{s} \dot{\boldsymbol{\phi}}_{l} \cdot \mathbf{S}_{l}^{b} \dot{\boldsymbol{\phi}}_{l}\right) \\
D^{\mathrm{in}}[\mathbf{u}] & =\int_{\Omega_{0}} \eta^{\mathrm{in}}\left|\nabla_{s} \mathbf{u}\right|^{2} d a=R \sum_{l=0}^{\infty} \eta^{\mathrm{in}} \dot{\boldsymbol{\phi}}_{l} \cdot \mathbf{D}_{l}^{\mathrm{in}} \dot{\boldsymbol{\phi}}_{l} \\
D^{\mathrm{ex}}[\mathbf{u}] & =\int_{\mathbb{R}^{3} \backslash \Omega_{0}} \eta^{\mathrm{ex}}\left|\nabla_{s} \mathbf{u}\right|^{2} d a=R \sum_{l=0}^{\infty} \eta^{\mathrm{ex}} \dot{\boldsymbol{\phi}}_{l} \cdot \mathbf{D}_{l}^{\mathrm{ex}} \dot{\boldsymbol{\phi}}_{l} .
\end{aligned}
$$


In addition, different modes evolve independently since the system is linear, and hence the energy balance equation (37) restricted to a single mode $l$ implies the momentum balance equation (47). From this viewpoint, the system is completely specified in terms of boundary displacement and velocity $\phi_{l}, \dot{\phi}_{l}$, and functionals:

$$
H\left[\boldsymbol{\phi}_{l}\right]=\frac{1}{2} \sum_{l=0}^{\infty} \boldsymbol{\phi}_{l} \cdot \mathbf{S}_{l} \boldsymbol{\phi}_{l}, \quad D\left[\boldsymbol{\phi}_{l}\right]=\sum_{l=0}^{\infty} \dot{\phi}_{l} \cdot \mathbf{D}_{l} \dot{\boldsymbol{\phi}}_{l},
$$

where $H\left[\phi_{l}\right]$ (resp. $D\left[\phi_{l}\right]$ ) may be referred to as the Hamiltonian (resp. rate of dissipation) of the system. This viewpoint explains why the matrices in (48) are symmetric and semi-positive-definite, and is particularly convenient if alternative viscoelastic models are employed for the surface or the bulk.

Remark 9 (Alternative elastic models for the membrane). In (42) we have used the particular Gurtin-Murdoch model (9) for the elastic behaviors of the surface membrane. Alternative elastic models may be employed for the membrane. For example, if the surface tension model (18) or (20) is employed, by Remark 5, (27), and (A.9)-(A.10) the surface strain energy of the membrane can be written as

$$
E_{s}[\mathbf{u}]=\frac{\gamma}{2} \sum_{l=0}^{\infty} \boldsymbol{\phi}_{l} \cdot \mathbf{S}_{l}^{e} \boldsymbol{\phi}_{l}, \quad \mathbf{S}_{l}^{e}=\left[\begin{array}{cc}
\tilde{l}-2 & 0 \\
0 & 0
\end{array}\right]
$$

if the linearized Helfrich model (29) is employed, by Remark 6 and (A.11) the surface elastic energy of the membrane can be written as

$$
E_{s}[\mathbf{u}]=\frac{\kappa_{b}}{2 R^{2}} \sum_{l=0}^{\infty} \boldsymbol{\phi}_{l} \cdot \mathbf{S}_{l}^{f} \boldsymbol{\phi}_{l}, \quad \mathbf{S}_{l}^{f}=\left[\begin{array}{cc}
(1-\tilde{l} / 2)^{2} & 0 \\
0 & 0
\end{array}\right]
$$

We also recall that

$$
\begin{aligned}
& \mathbf{D}_{l}=\eta^{\text {in }} R \mathbf{D}_{l}^{\text {in }}+\eta^{\text {ex }} R \mathbf{D}_{l}^{\text {ex }}+\eta_{s} \mathbf{S}_{l}^{a}+\chi_{s} \mathbf{S}_{l}^{b}=\eta^{\text {in }} R\left[\begin{array}{cc}
1+\frac{3}{l}+2 l & -3(1+l) \\
-3(l+1) & l(l+1)(2 l+1)
\end{array}\right] \\
& +\eta^{\operatorname{ex}} R\left[\begin{array}{cc}
\frac{2 l^{2}+3 l+4}{l+1} & -3 l \\
-3 l & l(l+1)(2 l+1)
\end{array}\right]+\eta_{s} \mathbf{S}_{l}^{a}+\chi_{s} \mathbf{S}_{l}^{b}
\end{aligned}
$$

and $\mathbf{K}_{l}=\mathbf{D}_{l}^{-1} \mathbf{S}_{l}$. For future convenience, we list below the matrices $\mathbf{S}_{l}$ for surface elasticity models discussed in Section 2.2.

1) Gurtin-Murdoch surface elasticity:

$$
\begin{aligned}
\mathbf{S}_{l}=2 \mu_{s} \mathbf{S}_{l}^{a}+\lambda_{s} \mathbf{S}_{l}^{b}+\mu_{b} R \mathbf{D}_{l}^{\text {in }}= & 2 \mu_{s}\left[\begin{array}{cc}
2 & -\tilde{l} \\
-\tilde{l} & \tilde{l}^{2}-\tilde{l}
\end{array}\right]+\lambda_{s}\left[\begin{array}{cc}
4 & -2 \tilde{l} \\
-2 \tilde{l} & \tilde{l}^{2}
\end{array}\right] \\
& +\mu_{b} R\left[\begin{array}{cc}
\left(1+\frac{3}{l}+2 l\right) & -3(1+l) \\
-3(l+1) & l(l+1)(2 l+1)
\end{array}\right] .
\end{aligned}
$$



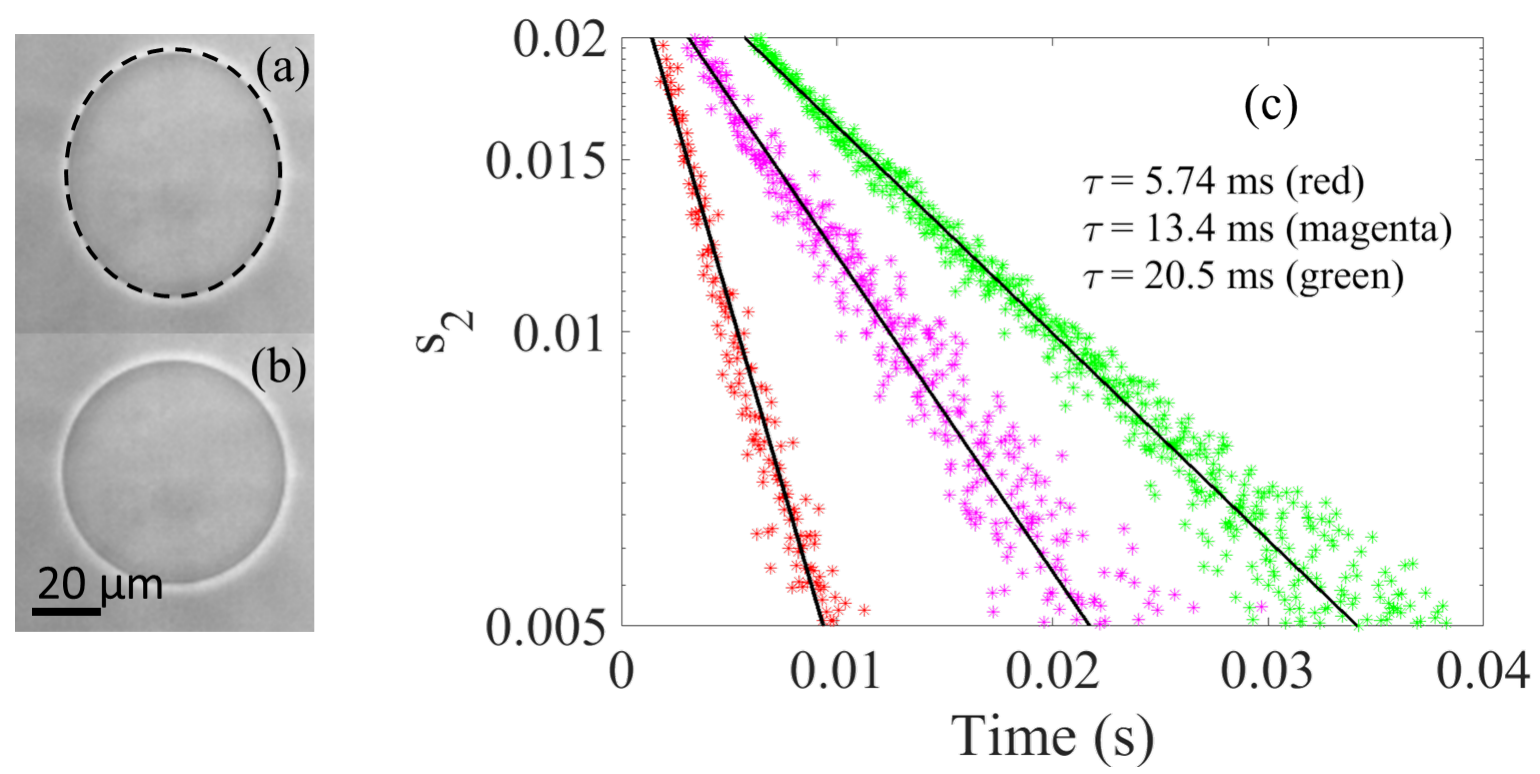

Figure 2: Deformation and relaxation of vesicles: (a) The vesicle deforms into a spheroidal shape upon electrodeformation. (b) The vesicle relaxes back to a perfect sphere in typically tens of milliseconds upon removal of the electric field. (c) The amplitudes of the $l=2$ mode (spheroidal mode) versus time in the quasi-spherical regime $\left(s_{2}<0.02\right)$ in three relaxation experiments. The curve exhibits a single-exponential decaying behavior as predicted by (68). The corresponding timescales are given in the figure.

\section{2) Linearized constant surface-tension membrane:}

$$
\mathbf{S}_{l}=\mu_{b} R \mathbf{D}_{l}^{\text {in }}+\gamma \mathbf{S}_{l}^{e}=\left[\begin{array}{cc}
\gamma(\tilde{l}-2)+\mu_{b} R\left(1+\frac{3}{l}+2 l\right) & -3 \mu_{b} R(1+l) \\
-3 \mu_{b} R(l+1) & \mu_{b} R l(l+1)(2 l+1)
\end{array}\right] .
$$

3) Linearized Helfrich membrane:

$$
\mathbf{S}_{l}=\mu_{b} R \mathbf{D}_{l}^{\text {in }}+\frac{4 \kappa_{b}}{R^{2}} \mathbf{S}_{l}^{f}=\left[\begin{array}{cc}
\frac{4 \kappa_{b}}{R^{2}}(1-\tilde{l} / 2)^{2}+\mu_{b} R\left(1+\frac{3}{l}+2 l\right) & -3 \mu_{b} R(1+l) \\
-3 \mu_{b} R(l+1) & \mu_{b} R l(l+1)(2 l+1)
\end{array}\right] .
$$

From (52) or (53), we see that the matrices $\mathbf{D}_{l}$ and $\mathbf{S}_{l}$ have to be positive definite in generic cases, and hence the matrices $\mathbf{K}_{l}=\mathbf{D}_{l}^{-1} \mathbf{S}_{l}$ can be diagonalized as

$$
\mathbf{K}_{l}=\mathbf{D}_{l}^{-1} \mathbf{S}_{l}=\mathbf{P}_{l} \operatorname{diag}\left[\frac{1}{\tau_{l}^{1}}, \frac{1}{\tau_{l}^{2}}\right] \mathbf{P}_{l}^{-1},
$$

where $\mathbf{P}_{l} \in \mathbb{R}^{2 \times 2}$ is a nonsingular matrix and $0<\tau_{l}^{1} \leq \tau_{l}^{2}$ represent two time-scales that can be measured by experiments. From expressions (56)-(59) of matrices $\mathbf{D}_{l}$ and $\mathbf{S}_{l}$, the precise dependence of two time-scales $\tau_{l}^{1}, \tau_{l}^{2}$ and matrix $\mathbf{P}_{l}$ on the viscoelastic properties can be explicitly calculated once the surface elasticity model is specified. 


\subsection{Applications of the explicit solutions}

As shown in Figs. 2-3, we can experimentally deform and monitor a cell or cell-aggregate. Our goal is to obtain the viscoelastic properties (i.e., $\mu_{b}, \gamma, \kappa_{b}, \eta^{\mathrm{in}}, \eta^{\mathrm{ex}}, \eta_{s}, \chi_{s}$ ) of the bulk and surface that can be used for quantifying the physiological state of cells or examining the efficacy of drugs in mitigating the dispersal of tumor cells (Shannon et al., 2015). In a deformation and relaxation process, we can obtain the radial displacement and its dependence on time $t$, i.e., $X_{l}=X_{l}(t)$ in (46), by processing optical imaging data. From the explicit solutions (49)-(51) and the expressions (56)-(60) of matrices of $\mathbf{D}_{l}, \mathbf{S}_{l}$ and $\mathbf{K}_{l}$, we find some characteristic behaviors of $X_{l}=X_{l}(t)$ that will be useful for extracting the viscoelastic properties of the system.

Deformation under constant loadings. If the body is deformed from its natural state (i.e., $\phi(0)=0$ ) by a time-independence surface traction $\mathbf{f}_{l}$, by (50) and (60) we see that the radial displacement $X_{l}=X_{l}(t)$ has to be of form:

$$
X_{l}(t)=C_{0}\left(1-C_{1} e^{-t / \tau_{l}^{1}}-C_{2} e^{-t / \tau_{l}^{2}}\right)
$$

where $C_{i}(i=0,1,2)$ are real constants and $C_{1}+C_{2}=1$. The experimentally measured radial displacement $X_{l}=X_{l}(t)$ can be used to fit with the above solution, determine the two time-scales $\tau_{l}^{1}, \tau_{l}^{2}$, and infer the viscoelastic properties of the system.

Phase-shift under sinusoidal loadings. If the body is deformed from its natural state (i.e., $\phi(0)=0$ ) by a sinusoidal surface traction $\mathbf{f}_{l}(t)=\mathbf{f}_{l}^{0} e^{i \omega t}$, by (51) and (60) we see that the radial displacement $X_{l}=X_{l}(t)$ has to be of form if $t \gg 1$ :

$$
X_{l}(t)=C_{0} e^{i(\omega t+\theta(\omega))}, \quad \theta(\omega)=\operatorname{Arg}\left(C_{1} \frac{\tau_{l}^{1}}{1+i \omega \tau_{l}^{1}}+C_{2} \frac{\tau_{l}^{2}}{1+i \omega \tau_{l}^{2}}\right)
$$

where $C_{i}(i=0,1,2)$ are real constants. The phase-shift $\theta(\omega)$ can be measured by comparing the forcing signals and displacement signals. From its frequency dependence, by (62) we can again determine the two time-scales $\tau_{l}^{1}, \tau_{l}^{2}$ and infer the viscoelastic properties of the system.

Relaxation. If the deformed quasi-spherical body is relaxed, i.e., $\boldsymbol{\phi}_{l}(0) \neq 0$ but $\mathbf{f}_{l}=0$, by (50) and (60) we see that the radial displacement $X_{l}=X_{l}(t)$ has to be of the form:

$$
X_{l}(t)=X(0)\left(C_{1} e^{-t / \tau_{l}^{1}}+C_{2} e^{-t / \tau_{l}^{2}}\right)
$$

where $C_{i}(i=1,2)$ are real constants and $C_{1}+C_{2}=1$. Again, the measured radial displacement $X_{l}=X_{l}(t)$ can be used to determine the two time-scales $\tau_{l}^{1}, \tau_{l}^{2}$ and infer the viscoelastic properties of the system.

As an example of applications, we first consider the experimental data on the relaxations of vesicles in $\mathrm{Yu}$ et al. (2015). As shown in Fig. 2(a)-(b), vesicles were first deformed by pulses of a DC electric field, then relaxed upon removal of the electric field. We focus on the leading mode of deformation, i.e., $l=2$, since the zeroth mode has to vanish because of incompressibility and the first mode corresponds to translation. We set $\mu_{b}=0$ because the 
medium inside the vesicle is also fluid, and select the constant surface tension model (27) for the surface membrane. By Remark 9, (56), (58), and neglecting the surface viscosity (i.e., $\left.\eta_{s}=\chi_{s}=0\right)$, we have that $(l=2)$

$$
\mathbf{D}_{2}=\eta^{\text {in }} R\left[\begin{array}{cc}
\frac{13}{2} & -9 \\
-9 & 30
\end{array}\right]+\eta^{\text {ex }} R\left[\begin{array}{cc}
6 & -6 \\
-6 & 30
\end{array}\right], \quad \mathbf{S}_{2}=\gamma\left[\begin{array}{cc}
4 & 0 \\
0 & 0
\end{array}\right]
$$

Therefore, for a relaxation process $\left(\mathbf{f}_{l}=0\right)$ the evolution equation (47) for the radial displacement $X_{2}=X_{2}(t)$ can be written as $\left(\hat{\eta}_{r}=\eta^{\text {in }} / \eta^{\text {ex }}\right)$

$$
\frac{38 \hat{\eta}_{r}^{2}+89 \hat{\eta}_{r}+48}{40\left(\hat{\eta}_{r}+1\right)} \dot{X}_{2}+\frac{\gamma}{R \eta^{\mathrm{ex}}} X_{2}=0
$$

which implies an exponential relaxation behavior

$$
X_{2}(t)=X_{2}(0) e^{-t / \tau}, \quad \tau=\frac{38 \hat{\eta}_{r}^{2}+89 \hat{\eta}_{r}+48}{4\left(10 \hat{\eta}_{r}+10\right)} \frac{R \eta^{\mathrm{ex}}}{\gamma} .
$$

We remark that the above solution recovers the relaxation behavior of fluid droplets (Zhang et al., 2013).

On the other hand, from the assumption that the vesicle membrane is not stretchable, Yu et al. (2015) enforce a local differential constraint (cf., Remark 2):

$$
\operatorname{div}_{s} \mathbf{u}=0 \quad \text { on } \partial B_{R}
$$

which, by (A.6) ${ }_{3}$ in Appendix A, implies that

$$
2 X_{l}=\tilde{l} \Theta_{l}
$$

Therefore, in terms of $X_{l}$ the total rate of energy dissipation of the exterior and interior flows is given by $X_{l}^{2}[1,2 / \tilde{l}] \cdot \mathbf{D}_{l}\left[1,2 / \tilde{l}^{T}\left(=\left(32 \eta^{\text {ex }}+23 \eta^{\text {in }}\right) X_{2}^{2} / 6\right.\right.$ for $\left.l=2\right)$, and hence by $(64)$ the evolution equation for $X_{2}$ shall be

$$
\frac{32+23 \hat{\eta}_{r}}{24} \dot{X}_{2}+\frac{\gamma}{R \eta^{\mathrm{ex}}} X_{2}=0
$$

implying a relaxation behavior different from (66):

$$
X_{2}(t)=X_{2}(0) e^{-t / \tau}, \quad \tau=\frac{32+23 \hat{\eta}_{r}}{24} \frac{R \eta^{\mathrm{ex}}}{\gamma} .
$$

An exemplary set of measurements is shown in Fig. 2(c). As shown in Fig. 2(c) $\left(s_{l} \propto\right.$ $X_{l}$ ), we see that the radial displacement $X_{2}$ indeed exhibits a single exponential decaying behavior as predicted by (68). Upon fitting (68) with the experimental data, we determine the relaxation time-scale $\tau$ as shown in Fig. 2(c), i.e., a relationship between the viscoelastic property of the membrane and viscous property of interior or exterior fluids. More data, experimental setup and their implications have been presented in Yu et al. (2015). 
We remark that the surface tension of a vesicle in general depends on deformation
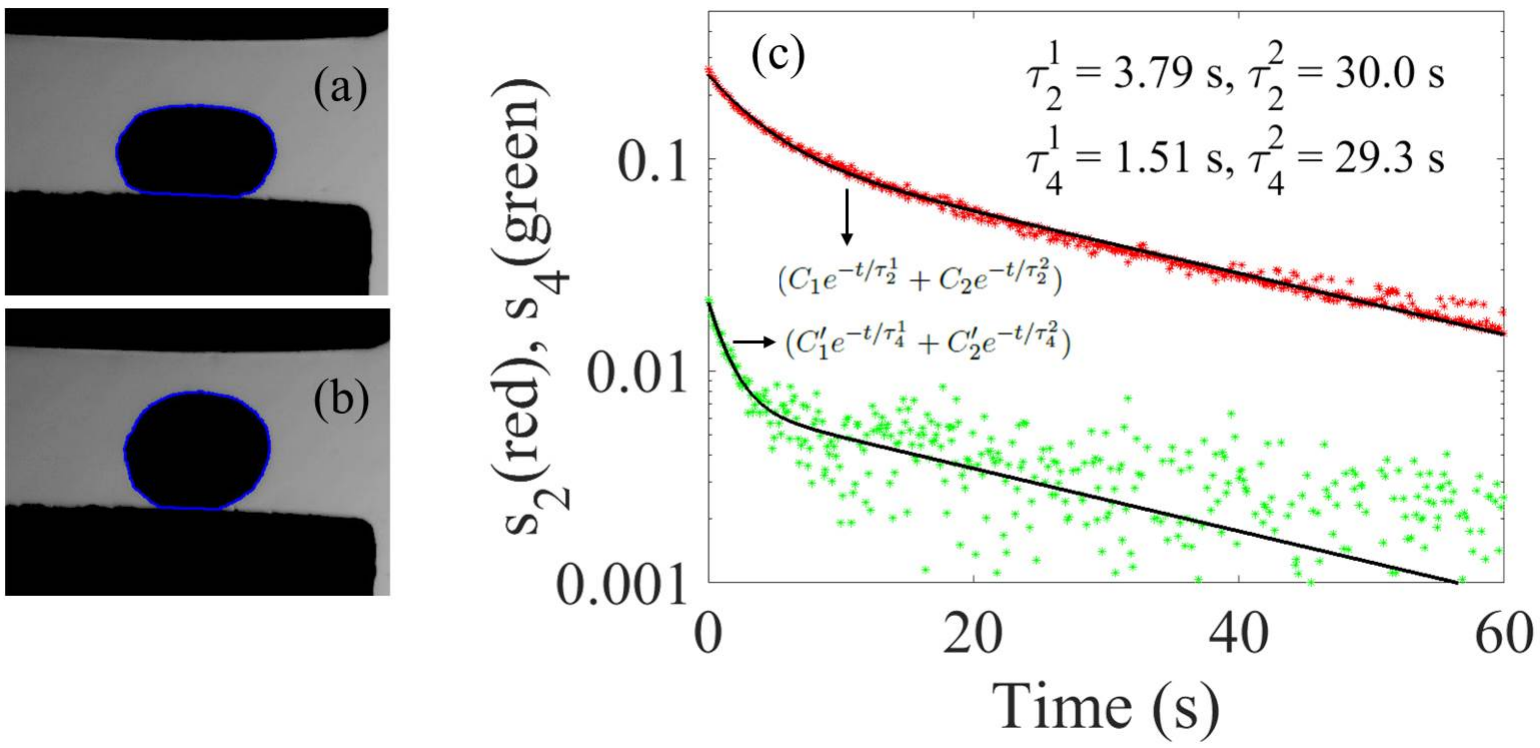

Figure 3: Deformation and relaxation of a cell aggregate: (a) Shape of the cell aggregate immediately after the removal of the indenter. (b) Shape of the cell aggregate after two minutes. The blue contour is generated for every image frame in order to compute the relaxation curve. (c) The amplitude of the deformation modes $(l=2,4)$ versus time. Fitting the curves with $(63)$ determines four relaxation time-scales: $\tau_{2}^{1}=3.79$ $\mathrm{s}, \tau_{2}^{2}=30.0 \mathrm{~s}, \tau_{4}^{1}=1.51 \mathrm{~s}$, and $\tau_{4}^{2}=29.3 \mathrm{~s}$.

A second set of experiments concern cell aggregates as shown in Fig. 3. In this case, we expect the interior body has nontrivial bulk elasticity. Again we neglect the surface viscosity (i.e., $\left.\eta_{s}=\chi_{s}=0\right)$. By (56) and (58), we have that for $l=2$ :

$$
\mathbf{D}_{2}=\eta^{\text {in }} R\left[\begin{array}{cc}
\frac{13}{2} & -9 \\
-9 & 30
\end{array}\right]+\eta^{\text {ex }} R\left[\begin{array}{cc}
6 & -6 \\
-6 & 30
\end{array}\right], \quad \mathbf{S}_{2}=\gamma\left[\begin{array}{cc}
4 & 0 \\
0 & 0
\end{array}\right]+\mu_{b} R\left[\begin{array}{cc}
\frac{13}{2} & -9 \\
-9 & 30
\end{array}\right]
$$

Therefore, the matrix $\mathbf{K}_{2}=\mathbf{D}_{2}^{-1} \mathbf{S}_{2}$ is given by

$$
\mathbf{K}_{2}=\frac{1}{48+89 \hat{\eta}_{r}+38 \hat{\eta}_{r}^{2}}\left[\begin{array}{cc}
40\left(1+\hat{\eta}_{r}\right) \frac{\gamma}{R \eta^{\mathrm{ex}}}+\left(47+38 \hat{\eta}_{r}\right) \frac{\mu_{b}}{\eta^{\mathrm{ex}}} & -30 \frac{\mu_{b}}{\eta^{\mathrm{ex}}} \\
\left(8+12 \hat{\eta}_{r}\right) \frac{\gamma}{R \eta^{\mathrm{ex}}}-5 \frac{\mu_{b}}{\eta^{\mathrm{ex}}} & \left(42+38 \hat{\eta}_{r}\right) \frac{\mu_{b}}{\eta^{\mathrm{ex}}}
\end{array}\right] .
$$

The two eigenvalues of $\mathbf{K}_{2}$ (denoted by $1 / \tau_{2}^{1}, 1 / \tau_{2}^{2}$ ) can clearly be explicitly calculated; the expressions are however too tedious to be presented here. In the case that the exterior viscosity is negligible, i.e., $\eta^{\mathrm{ex}}=0$, the two time-scales are simply given by

$$
\tau_{2}^{1}=\frac{\eta^{\text {in }} / \mu_{b}}{20 \gamma /\left(19 \mu_{b} R\right)+1}, \quad \tau_{2}^{2}=\frac{\eta^{\text {in }}}{\mu_{b}} .
$$

Similar calculations can be done for $l=4$, yielding two time-scales given by

$$
\tau_{4}^{1}=\frac{\eta^{\text {in }} / \mu_{b}}{36 \gamma /\left(17 \mu_{b} R\right)+1}, \quad \tau_{4}^{2}=\frac{\eta^{\text {in }}}{\mu_{b}}
$$


In other words, the evolution of radial displacement shall satisfy

$$
X_{2}(t)=X_{2}(0)\left(C_{1} e^{-t / \tau_{2}^{1}}+C_{2} e^{-t / \tau_{2}^{2}}\right), \quad X_{4}(t)=X_{4}(0)\left(C_{1}^{\prime} e^{-t / \tau_{4}^{1}}+C_{2}^{\prime} e^{-t / \tau_{4}^{2}}\right) .
$$

As shown in Fig. $3\left(s_{l} \propto X_{l}\right)$, we fit the experimental data with the above equations for $l=2,4$ and obtain four relaxation time-scales: $\tau_{2}^{1}=3.79 \mathrm{~s}, \tau_{2}^{2}=30.0 \mathrm{~s}, \tau_{4}^{1}=1.51 \mathrm{~s}$, and $\tau_{4}^{2}=29.3 \mathrm{~s}$. In other words,

$$
\frac{\eta^{\text {in }}}{\mu_{b}} \approx 29.7 s, \quad \frac{\gamma}{\mu_{b} R} \approx 7.6 .
$$

Therefore, if the surface tension $\gamma$ is estimated by, e.g., the method in Foty et al. (1994), the relaxation time-scales (i.e., $\tau_{l}^{1}, \tau_{l}^{2}$ ) will fully determine the viscoelastic properties of the bulk.

Two remarks are in order here regarding the characteristics of our relaxation solutions and experimental data shown in Fig. 2-3. First, we have strictly restricted ourselves to the regime of small strain and strain rate and fully linearized boundary value problems. As illustrated by (63) we see that a generic relaxation solution persists on double exponential decays of radial displacement of each mode (or single exponential decays in some degenerated cases e.g. (66) or (68)). Therefore, this relaxation characteristics alone cannot differentiate surface viscoelastic models. In other words, experimental data in Fig. 2(c) or 3(c) neither favor the surface tension than, e.g., the Gurtin-Murdoch model or the Helfrich-Canham bending model, nor imply the surface viscosity is indeed negligible. Additional data and analyses are necessary to differentiate material models for the surface or the bulk. Also, our central result in this work is to provide a solution framework for quickly obtaining the relations between the relaxations/deformations characteristics and the material properties of postulated material models. Though simple material models are employed in analyzing experimental data shown in Fig. 2-3, we do not intent to conclude they are the "right" models for the vesicles or cell aggregates. However, if they were the "right" models, the experimental data would imply the relations (66), (68), or (71) between the surface tension and bulk viscoelastic properties.

\subsection{Indentation by a frictionless axis-symmetric indenter}

\subsubsection{Formulation and general solutions}

In this section we consider the processes of deformation in experiments of indentations. As illustrated in Fig. 4, an axis-symmetric indenter is initially in contact with the spherical body at the north pole. The indenter surface admits a parametrization $z=h(\theta)$. For simplicity, we make the following assumptions: (i) the friction between the indenter and membrane is negligible, (ii) the axis of symmetry of the indenter is aligned with that of sphere, and (iii) the boundary conditions on the sphere is symmetric about the equator plane. Our main interest is to find the functional relation between the applied force, the displacement, and the velocity of the indenter:

$$
F_{z}=F_{z}\left(z_{\text {in }}, \dot{z}_{\text {in }}\right)
$$


where $F_{z}$ is the indentation force, and $z_{\text {in }}$ (resp. $\left.\dot{z}_{\text {in }}\right)$ is the displacement (resp. velocity) of the indenter.

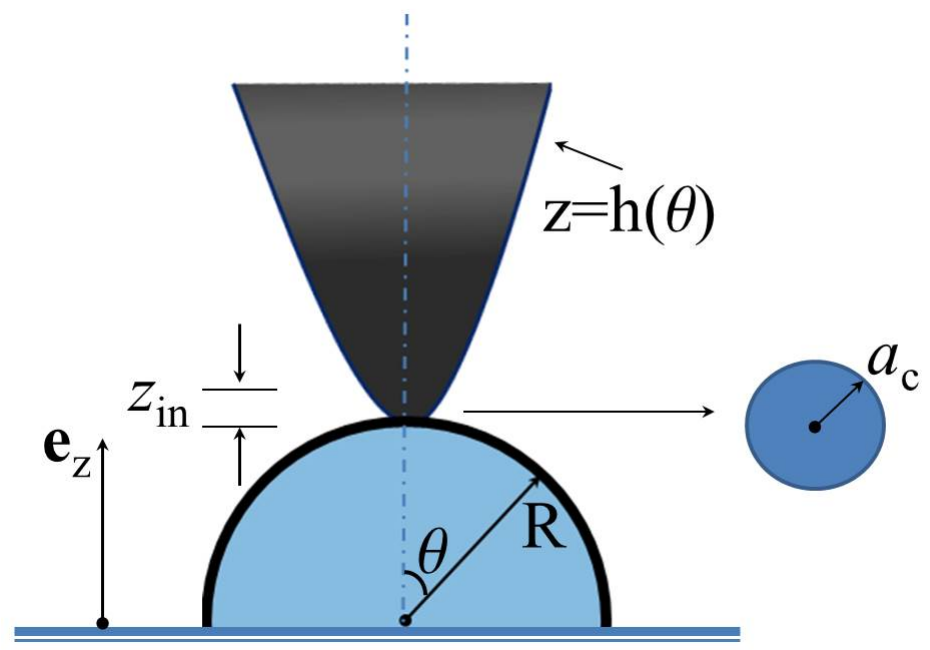

Figure 4: Indentation of a spherical viscoelastic body with surface viscoelasticity.

Because of axis-symmetry, the contact area remains disk-shaped. As illustrated in Fig. 4, we denote by $a_{c}(t)$ the radius of the contact area, by $\theta_{c}=a_{c} / R \ll 1$ the corresponding angle, by

$$
\mathcal{A}_{c}=\left\{(R, \theta, \varphi): \theta \in\left[0, \theta_{c}\right], \varphi \in[0,2 \pi]\right\}
$$

the contact area on the reference sphere $\partial \Omega_{0}$, and by

$$
\mathbf{t}^{s}=\frac{a_{c} q(\theta, t)}{2 R \sin \theta} \hat{\mathbf{r}} \quad \text { if } \theta \in\left[0, \theta_{c}\right] \quad \text { and } \quad=0 \text { if } \theta \in\left(\theta_{c}, \pi / 2\right]
$$

the unknown surface traction due to the indenter. We note that the quantity $q(\theta, t)$ can be interpreted as the applied force per unit area and satisfy $q(\theta, t)=q(\pi-\theta, t)$ for being symmetric about the equator plane.

By (44)-(46), and symmetry, we write the displacements on $\partial B_{R}$ as

$$
\begin{aligned}
& \mathbf{u}(R, \theta)=u^{r}(\theta) \hat{\mathbf{r}}+u^{\theta}(\theta) \mathbf{e}_{\theta}, \\
& u^{r}(\theta)=\sum_{l \text { even }} X_{l} Y_{l}(\theta), \quad u^{\theta}(\theta)=\sum_{l \text { even }} \Theta_{l} Y_{l}^{\prime}(\theta) .
\end{aligned}
$$

By Remark 8, the traction on the boundary $\partial B_{R}$ is given by

$$
\left.\left[\begin{array}{c}
\sigma^{r r}(r, \theta, t) \\
\sigma^{r \theta}(r, \theta, t)
\end{array}\right]\right|_{r=R}=\sum_{l \text { even }}\left(\mathbf{S}_{l}\left[\begin{array}{c}
X_{l}(t) Y_{l}(\theta) \\
\Theta_{l}(t) Y_{l}^{\prime}(\theta)
\end{array}\right]+\mathbf{D}_{l} \frac{d}{d t}\left[\begin{array}{c}
X_{l}(t) Y_{l}(\theta) \\
\Theta_{l}(t) Y_{l}^{\prime}(\theta)
\end{array}\right]\right) .
$$


It will be convenient to write the matrices $\mathbf{S}_{l}$ and $\mathbf{D}_{l}$ in (47) as

$$
\mathbf{S}_{l}=\left[\begin{array}{cc}
S_{l}^{r r} & S_{l}^{r \theta} \\
S_{l}^{r \theta} & S_{l}^{\theta \theta}
\end{array}\right], \quad \mathbf{D}_{l}=\left[\begin{array}{cc}
D_{l}^{r r} & D_{l}^{r \theta} \\
D_{l}^{r \theta} & D_{l}^{\theta \theta}
\end{array}\right]
$$

where the values of components of $\mathbf{S}_{l}$ and $\mathbf{D}_{l}$ have been explicitly calculated in Remark 9, (56)-(59). For a frictionless indenter, the boundary conditions at $r=R$ include the following:

$$
\begin{aligned}
& \text { (i) } \quad \sigma^{r r}=\mathbf{t}^{s} \cdot \hat{\mathbf{r}} \text { if } \theta \in\left[0, \theta_{c}\right] ; \quad=0 \text { if } \theta \in\left[\theta_{c}, \frac{\pi}{2}\right] ; \quad \sigma^{r \theta}=0 \text { if } \theta \in\left[0, \frac{\pi}{2}\right] \text {; } \\
& \text { (ii) } u^{r} \cos \theta-u^{\theta} \sin \theta=z_{\text {in }}(t)+h(\theta)-R \cos \theta \quad \text { if } \theta \in\left[0, \theta_{c}\right], \quad \text { or } \\
& (i i)^{\prime} u^{r}=z_{\text {in }}(t)+h(\theta)-R \quad \text { if } \theta \in\left[0, \theta_{c}\right] \text {, }
\end{aligned}
$$

where $(i i)^{\prime}$ is a good approximation of $(i i)$ since $\theta_{c} \ll 1$.

The total applied force on the upper hemisphere is along the $z$-direction and given by (cf., (73)-(74))

$$
F_{z}(t)=\int_{\mathcal{A}_{c}} \mathbf{t}^{s} \cdot \mathbf{e}_{z} d a=\pi R a_{c} \int_{0}^{\theta_{c}} q(\theta, t) \cos \theta d \theta \approx \pi R a_{c} \int_{0}^{\theta_{c}} q(\theta, t) d \theta .
$$

From the definition (44) and (A.5), we have

$$
t_{l}^{r}(t)=\frac{1}{R^{2}} \int_{\partial \Omega_{0}} \hat{\mathbf{r}} \cdot \mathbf{t}^{s}(\theta, t) Y_{l}(\theta) d a= \begin{cases}0 & \text { if } l \text { odd } \\ \frac{2 \pi a_{c}}{R} \int_{0}^{\theta_{c}} q(\theta, t) Y_{l}(\theta) d \theta & \text { if } l \text { even }\end{cases}
$$

Therefore, by (49) we obtain

$$
\phi_{l}(t)=\exp \left(-t \mathbf{K}_{l}\right)\left[\boldsymbol{\phi}_{l}(0)+R^{2} \int_{0}^{t} \exp \left(\tau \mathbf{K}_{l}\right) \mathbf{D}_{l}^{-1} \mathbf{f}_{l}(\tau) d \tau\right], \quad \mathbf{f}_{l}=\left[\begin{array}{c}
t_{l}^{r} \\
0
\end{array}\right]
$$

Inserting (80) into (76), the boundary conditions (77) imply a set of equations relating $q(\theta, t)$, $z_{\text {in }}$ and $\dot{z}_{\text {in }}(t)$, by which one can solve for $z_{\text {in }}(t)$ upon specifying $F_{z}(t)$ and vice versa. Below we consider a few typical scenarios that are useful for experimental measurements.

\subsubsection{Quasi-static solutions}

For quasi-static loadings, the velocity is small and the effect of viscosity may be neglected. In this case, the general solution to (47) is simply given by

$$
\phi_{l}=\left[\begin{array}{c}
X_{l} \\
\Theta_{l}
\end{array}\right]=R^{2} \mathbf{S}_{l}^{-1} \mathbf{f}_{l}=\frac{R^{2} t_{l}^{r}}{\operatorname{det} \mathbf{S}_{l}}\left[\begin{array}{c}
S_{l}^{\theta \theta} \\
-S_{l}^{r \theta}
\end{array}\right] .
$$

In particular, the radial displacement $u^{r}(R, \theta)$ can be written as

$$
u^{r}(R, \theta)=\sum_{l \text { even }} X_{l}(R) Y_{l}(\theta)=\int_{0}^{\theta_{c}} q\left(\theta^{\prime}\right) G\left(\theta, \theta^{\prime} ; R\right) d \theta^{\prime}
$$



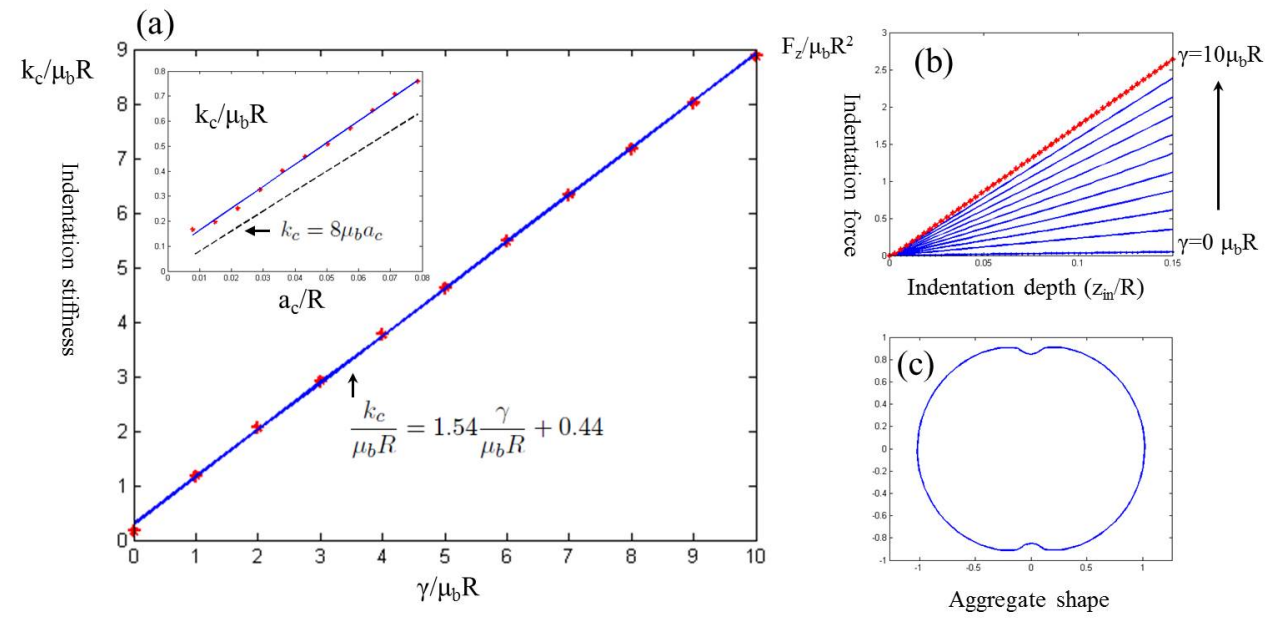

Figure 5: Indentation by a flat indenter of radius $a_{c}=R * \pi / 200$. (a) $k_{c}=k_{c}(\gamma)$ : Indentation stiffness $k_{c}$ versus surface tension $\gamma$. The discrete '*'-points are numerical results and the fitted continuous line show that the contact stiffness is an (approximate) linear function of surface tension. The inset shows the contact stiffness $k_{c}$ versus contact radius $a_{c}$ at $\gamma=0$. The dashed line '- -' represents the classic solution of flat-head indenter on an elastic half-space. (b) $F_{z}=F_{z}\left(z_{\mathrm{in}} ; \gamma\right)$ : Indentation force $F_{z}$ versus indentation depth $z_{\mathrm{in}}$ for varying surface tension $\gamma$. (c) Typical deformed shape of the spherical body $\left(\gamma=10 \mu_{b} R, z_{\text {in }}=0.15 R\right.$, $\left.a_{c}=R * \pi / 200\right)$.

where

$$
G\left(\theta, \theta^{\prime} ; R\right)=\sum_{l \text { even }} 2 \pi a R \alpha_{l} Y_{l}\left(\theta^{\prime}\right) Y_{l}(\theta), \quad \alpha_{l}:=\frac{S_{l}^{\theta \theta}}{\operatorname{det} \mathbf{S}_{l}}
$$

Then by the boundary condition (77), we can determine the contact radius $a_{c}$ and the forcedisplacement relations $F_{z}=F_{z}\left(z_{\text {in }}\right)$, which can then be used for probing elastic properties of the bulk and surface.

The quasi-static problem can be alternatively formulated as a variational inequality which will be particularly convenient for systematic approximate solutions and numerical solutions. By the principle of minimum free energy, the equilibrium state of the system shall be determined by

$$
\begin{aligned}
E_{\text {min }}\left(z_{\text {in }}\right): & =\min _{\left(u^{r}, u^{\theta}\right) \in \mathbb{K}}\left\{E\left[u^{r}, u^{\theta}\right]=\sum_{l \text { even }} \frac{1}{2} \phi_{l} \cdot \mathbf{S}_{l} \boldsymbol{\phi}_{l}\right\}, \\
\mathbb{K} & =\left\{\left(u^{r}, u^{\theta}\right): u^{r} \cos \theta-u^{\theta} \sin \theta \leq z_{\text {in }}+h(\theta)-R \cos \theta \quad \forall \theta \in\left[0, \frac{\pi}{2}\right]\right\},
\end{aligned}
$$

where the constraints on the admissible space $\mathbb{K}$ arises from that the elastic surface cannot penetrate into the indenter and the inequality shall hold as an equality within the contact area, see (77) (ii). Below we present explicit approximate solutions for indenters of specific geometries.

Flat-headed indenter. If the indenter is flat-headed (i.e., $h(\theta)=R$ ) with a radius $a_{0},\left|u_{z}\right| \gg a_{0}$, and $a_{0} \ll R$, then the contact radius $a_{c}=a_{0}$ is constant. Since $\theta_{c} \ll 1$, we can safely neglect higher-order terms in the boundary condition (77)(ii) and enforce the 
approximate boundary condition:

$$
u^{r}=z_{\text {in }} \quad \forall \theta \in\left[0, \theta_{c}\right] .
$$

By (82) or (84), one can see that force-displacement relation is linear $F_{z}=k_{c} z_{\text {in }}$, or equivalently,

$$
E_{\min }\left(u_{z}\right)=2 \times \frac{1}{2} k_{c} z_{\text {in }}^{2}
$$

where the factor 2 arises from the symmetry, i.e., the bottom indenter.

The exact value of contact stiffness $k_{c}$ in general has to be numerically found by solving (82) or (84). In the Appendix C, we outline a simple numerical algorithm based on the variational inequality formulation (84). Closed-form approximate solutions can be obtained by keeping only the leading terms in the expressions of the elastic energy in (84) and enforcing the constraint (85) at some discrete points. For example, we may enforce (85) at only two points $\theta=0, \theta_{c}$ :

$$
\left.u^{r}\right|_{\theta=0}=z_{\text {in }},\left.\quad u^{r}\right|_{\theta=\theta_{c}}=z_{\text {in }},
$$

and consider only the modes $l=2,4$ in the elastic energy in (84). Then the minimization problem (84) is reduced to

$$
E_{\min }^{(4)}\left(z_{\text {in }}\right):=\min \left\{\frac{1}{2}\left(\phi_{2} \cdot \mathbf{S}_{2} \phi_{2}+\phi_{4} \cdot \mathbf{S}_{4} \boldsymbol{\phi}_{4}\right):\left.\left[X_{2} Y_{2}(\theta)+X_{4} Y_{4}(\theta)\right]\right|_{\theta=0, \theta_{c}}=z_{\text {in }}\right\} .
$$

The above algebraic minimization problem can be explicitly solved and the results are given by $\left(X_{2}, X_{4}\right)=z_{\text {in }}\left(\hat{X}_{2}, \hat{X}_{4}\right)$,

$$
\hat{X}_{2}=\frac{Y_{4}\left(\theta_{c}\right)-Y_{4}(0)}{Y_{2}(0) Y_{4}\left(\theta_{c}\right)-Y_{4}(0) Y_{2}\left(\theta_{c}\right)}, \quad \hat{X}_{4}=\frac{-Y_{2}\left(\theta_{c}\right)+Y_{2}(0)}{Y_{2}(0) Y_{4}\left(\theta_{c}\right)-Y_{4}(0) Y_{2}\left(\theta_{c}\right)},
$$

and

$$
E_{\min }^{(4)}\left(z_{\text {in }}\right)=k_{c}^{(4)} z_{\text {in }}^{2}, \quad k_{c}^{(4)}=\frac{\hat{X}_{2}^{2}}{2 \alpha_{2}}+\frac{\hat{X}_{4}^{2}}{2 \alpha_{4}}
$$

where $k_{c}^{(4)}$, by (86), can be interpreted as the approximate contact stiffness. We remark that the above approximate contact stiffness may be systematically improved by including more terms in the elastic energy in (84) and enforcing the constraint (85) at more points. More trustworthy results can be obtained by directly solving the variational inequality (84) by the numerical method described in Appendix C. For example, we consider the case $R=1$, $a_{c}=R * \pi / 200, \mu_{b}=1$, and $\gamma / \mu_{b} R=0,1, \cdots, 10$. The numerical results are illustrated in Fig. 5. From Fig. 5(b) we see that the indentation force indeed depends linearly on the indentation depth. Interestingly, from Fig. 5(a) we find that the indentation stiffness is roughly a linear function of the surface tension $\gamma$ within the regime of computation and 
given by

$$
\frac{k_{c}}{\mu_{b} R} \approx 1.54 \frac{\gamma}{\mu_{b} R}+0.44
$$

We remark that the above empirical linear dependence of contact stiffness on surface tension can be potentially useful for experimental measurements, though it does not rigorously follow from the solutions to (84). Moreover, if $\mu_{b} R \gg \gamma$ and $R \gg a_{0}$, we anticipate that the bulk elasticity dominates and the displace-force relation is given by the classic solution of flatheaded indenter in contact with an elastic half-space (Bower, 2009):

$$
k_{c}=8 \mu_{b} a_{c} .
$$

As shown in the inset in Fig. 5(a), we plot the contact stiffness $k_{c}$ versus contact radius $a_{c}$ ('*'-line) and the fitted solid line is given by

$$
\frac{k_{c}}{\mu_{b} R} \approx 8.7 \frac{a_{c}}{R}+0.07
$$

which agrees with (89) ('- -' line ) reasonably well. The minor discrepancy likely arises from the truncation of the elastic energy (C.26) and the body being finite (instead of an infinite half-space).
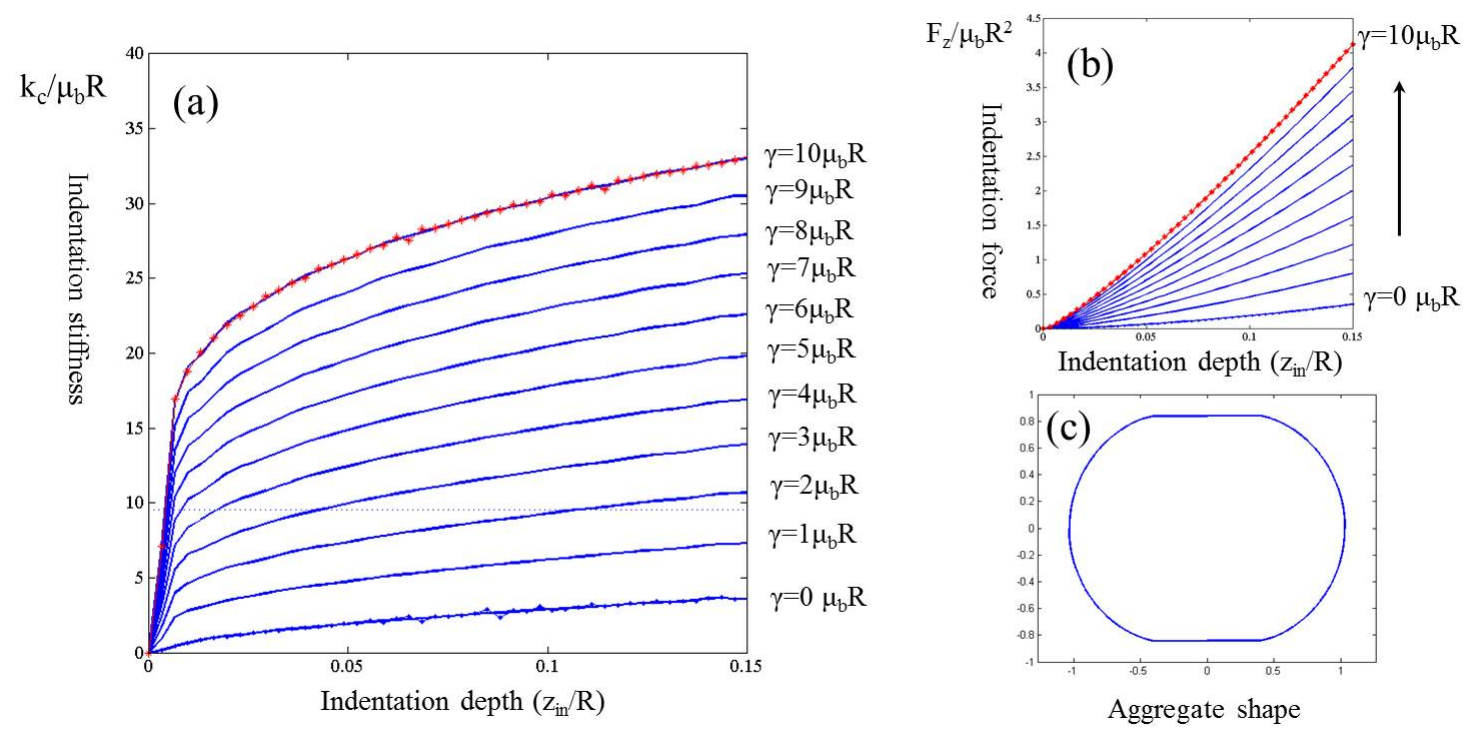

Figure 6: Indentation by a rigid plate. (a) $k_{c}=k_{c}\left(z_{\mathrm{in}} ; \gamma\right)$ : Contact stiffness $k_{c}$ versus indentation depth $z_{\text {in }}$ for varying surface tension $\gamma ;(\mathrm{b}) F_{z}=F_{z}\left(z_{\mathrm{in}} ; \gamma\right)$ : Indentation force $F_{z}$ versus indentation depth $z_{\text {in }}$ for varying surface tension $\gamma$; (c) Typical deformed shape of the spherical body $\left(\gamma=10 \mu_{b} R, z_{\text {in }}=0.15 R\right)$.

Compression between two rigid plates. If the indenter is an infinite rigid plate, the contact radius $a_{c}$ depends on the indentation depth $z_{\text {in }}$, implying a nonlinear relation $F_{z}=F_{z}\left(z_{\text {in }}\right)$. By the numerical method described in Appendix C, we explore the case $R=1$, $\mu_{b}=1$, and $\gamma / \mu_{b} R=0,1, \cdots, 10$. Figure $6\left(\right.$ a) shows the contact stiffness $k_{c}=\partial F_{z}\left(z_{\text {in }}\right) / \partial z_{\text {in }}$ 
for different surface tensions whereas Figure $6(\mathrm{~b})$ shows the force-displacement relation $F_{z}=$ $F_{z}\left(z_{\text {in }}\right)$. As expected, both plots reveal a nonlinear relationship between the indentation force $F_{z}$ and indentation depth $z_{\text {in }}$. Moreover, typical deformed body compressed by two rigid plates are shown in Fig. 6(c). Numerical results as shown in Fig. 6(a)-(c) can be used for extracting elastic properties of the system.

\section{Summary and discussion}

In this work, we have considered a model of a $2 \mathrm{D}$ viscoelastic surface coupled with 3D viscoelastic bulks. We believe that this general model better describes the viscoelastic behaviors of cells and cell aggregates than the uncoupled 2D viscoelastic surface or the 3D viscoelastic model. Moreover, we have systematically linearized the elastic models for fluid membranes including the surface tension model and the Helfrich-Canham bending model. For quasi-spherical deformations, we have obtained explicit analytical and numerical solutions for the relaxation and deformation processes. The solutions have been applied to fit with the experimental data and extract the viscoelastic properties of the surface and bulk.

The central problem for a biological system is often about deciding what is the "right" material models for the system. We have therefore strived to establish a framework for generating analytical and numerical solutions for different surface and bulk viscoelastic models within the regime of small strains and quasi-spherical configurations. We anticipate that the current work will have interesting applications not only in extracting material properties, but also in selecting suitable material models for biological systems.

\section{Appendix}

\section{A. Spherical coordinates and spherical harmonics}

Let $(r, \theta, \varphi)$ be the the usual spherical coordinates. First, we recall that axis-symmetric spherical harmonics are defined as

$$
Y_{l}(\theta)=\sqrt{\frac{2 l+1}{4 \pi}} P_{l}(\cos \theta), \quad P_{l}(x)=\frac{1}{2^{l} l !} \frac{d^{l}}{d x^{l}}\left[\left(x^{2}-1\right)^{l}\right],
$$

where $P_{l}:(-1,1) \rightarrow \mathbb{R}$ is the Legendre polynomials satisfying

$$
\frac{d}{d x}\left[\left(1-x^{2}\right) \frac{d}{d x} P_{l}(x)\right]+l(l+1) P_{l}(x)=0
$$

Therefore, we have that $\left(\tilde{l}=l(l+1)\right.$ and $\left.{ }^{\prime}=d / d \theta\right)$

$$
r^{2} \Delta Y_{l}=Y_{l}^{\prime \prime}+\cot \theta Y_{l}^{\prime}=-\tilde{l} Y_{l},
$$


and the orthogonal properties ( $\mathbb{S}$ is the unit spherical surface):

$$
\begin{aligned}
& \int_{\mathbb{S}} Y_{l} Y_{k} d a=\delta_{l k}, \quad \int_{\mathbb{S}} Y_{l}^{\prime} Y_{k}^{\prime} d a=\tilde{l} \delta_{l k}, \quad \int_{\mathbb{S}}\left(\Delta_{s} Y_{l}\right)\left(\Delta_{s} Y_{k}\right) d a=(\tilde{l})^{2} \delta_{l k}, \\
& I_{l k}:=\int_{\mathbb{S}} Y_{l}^{\prime \prime} Y_{k}^{\prime} \cot \theta d a=-\int_{\mathbb{S}} Y_{l}^{\prime} Y_{k}^{\prime \prime} \cot \theta d a+\int_{\mathbb{S}} Y_{l}^{\prime} Y_{k}^{\prime} d a=-I_{k l}+\tilde{l} \delta_{k l}, \\
& \int_{\mathbb{S}}\left(Y_{l}^{\prime \prime} Y_{k}^{\prime \prime}+Y_{l}^{\prime} \cot \theta Y_{k}^{\prime} \cot \theta\right)=\int_{\mathbb{S}}\left(Y_{l}^{\prime \prime}+Y_{l}^{\prime} \cot \theta\right)\left(Y_{k}^{\prime \prime}+Y_{k}^{\prime} \cot \theta\right)-I_{l k}-I_{k l}=\left(\tilde{l}^{2}-\tilde{l}\right) \delta_{k l} .
\end{aligned}
$$

The axis-symmetric vector spherical harmonics are defined as $\left(\hat{\mathbf{r}}=\mathbf{r} / r, \mathbf{e}_{\theta}, \mathbf{e}_{\varphi}\right.$ are the unit basis vectors of spherical coordinate $(r, \theta, \varphi))$

$$
\mathbf{Y}_{l}=Y_{l} \hat{\mathbf{r}}, \quad \mathbf{\Psi}_{l}=r \nabla Y_{l}=Y_{l}^{\prime} \mathbf{e}_{\theta}, \quad \boldsymbol{\Phi}_{l}=\mathbf{r} \times \nabla Y_{l} .
$$

For brevity, we define operators:

$$
\mathcal{L}_{r}=\frac{\partial}{\partial r}+\frac{1}{r}, \quad \mathcal{L}_{r}^{2}=\frac{1}{r^{2}} \frac{\partial}{\partial} r^{2} \frac{\partial}{\partial r}, \quad \Delta_{s}=\Delta-\mathcal{L}_{r}^{2} .
$$

Consider an axis-symmetric vector field

$$
\mathbf{u}(\mathbf{x})=u^{r}(r, \theta) \hat{\mathbf{r}}+u^{\theta}(r, \theta) \mathbf{e}_{\theta}=\sum_{l=0}^{\infty}\left(X_{l}(r) \mathbf{Y}_{l}+\Theta_{l}(r) \mathbf{\Psi}_{l}\right)
$$

where by (A.2) we have

$$
X_{l}(r)=\frac{1}{r^{2}} \int_{\partial B_{r}} u^{r}(r, \theta) Y_{l}(\theta) d a, \quad \Theta_{l}(r)=\frac{1}{\tilde{l} r^{2}} \int_{\partial B_{r}} u^{\theta}(r, \theta) Y_{l}^{\prime}(\theta) d a .
$$

Since the surface gradient $\nabla_{s}$ on $\partial B_{r}$ can be written as

$$
\nabla_{s}=\frac{\partial}{r \partial \theta} \mathbf{e}_{\theta}+\frac{\partial}{r \sin \theta \partial \varphi} \mathbf{e}_{\varphi}
$$

we find that

$$
\begin{aligned}
& \nabla_{s} \mathbf{e}_{\theta}=\frac{1}{r}\left(-\hat{\mathbf{r}} \otimes \mathbf{e}_{\theta}+\cot \theta \mathbf{e}_{\varphi} \otimes \mathbf{e}_{\varphi}\right), \quad \nabla_{s} \mathbf{e}_{\varphi}=-\frac{1}{r \sin \theta}\left(\cos \theta \mathbf{e}_{\theta}+\sin \theta \hat{\mathbf{r}}\right) \otimes \mathbf{e}_{\varphi}, \\
& \nabla_{s} Y_{l}=r^{-1} Y_{l}^{\prime} \mathbf{e}_{\theta}=r^{-1} \Psi_{l}, \quad \nabla_{s} \nabla_{s} Y_{l}=r^{-2}\left[Y_{l}^{\prime \prime} \mathbf{e}_{\theta} \otimes \mathbf{e}_{\theta}-Y_{l}^{\prime} \hat{\mathbf{r}} \otimes \mathbf{e}_{\theta}+\cot \theta Y_{l}^{\prime} \mathbf{e}_{\varphi} \otimes \mathbf{e}_{\varphi}\right] .
\end{aligned}
$$


Therefore, for the vector field in (A.4) restricted to $\partial B_{r}$ we obtain that

$$
\begin{aligned}
& \nabla_{s} \mathbf{u}=\sum_{l=0}^{\infty}\left[X_{l} r^{-1}\left(\hat{\mathbf{r}} \otimes \mathbf{\Psi}_{l}+Y_{l} \mathbf{I}_{\nu}\right)+\Theta_{l} r \nabla_{s} \nabla_{s} Y_{l}\right], \\
& \mathbb{P}_{\nu}\left(\nabla_{s} \mathbf{u}\right)=\sum_{l=0}^{\infty}\left[X_{l} r^{-1} Y_{l} \mathbf{I}_{\nu}+\Theta_{l} r\left(\mathbb{P}_{\nu} \nabla_{s} \nabla_{s} Y_{l}\right)\right] \\
& \operatorname{Tr}\left[\mathbb{P}_{\nu}\left(\nabla_{s} \mathbf{u}\right)\right]=\nabla_{s} \cdot \mathbf{u}=\sum_{l=0}^{\infty}\left[2 X_{l}-\tilde{l} \Theta_{l}\right] r^{-1} Y_{l},
\end{aligned}
$$

and that

$$
\begin{aligned}
& \Delta_{s} \nabla_{s} Y_{l}=\Delta_{s} \nabla Y_{l}=\left(\Delta-\mathcal{L}_{r}^{2}\right) \nabla Y_{l}=\left(\nabla \Delta Y_{l}-\mathcal{L}_{r}^{2} \nabla Y_{l}\right) \\
& =-\tilde{l} \nabla \frac{Y_{l}}{r^{2}}-\left(r \nabla Y_{l}\right) \mathcal{L}_{r}^{2} \frac{1}{r}=r^{-3}\left[-\tilde{l} \mathbf{\Psi}_{l}+2 \tilde{l} \mathbf{Y}_{l}\right], \\
& \operatorname{div}_{s}\left[\mathbb{P}_{\nu}\left(\nabla_{s} \nabla_{s} Y_{l}\right)\right]=-r^{-1} \boldsymbol{\nu} \Delta_{s} Y_{l}+r^{-3} \mathbf{\Psi}_{l}+\mathbf{I}_{\nu}\left(\Delta_{s} \nabla_{s} Y\right)=r^{-3}\left(\tilde{l} \mathbf{Y}_{l}-(\tilde{l}-1) \mathbf{\Psi}_{l}\right), \\
& \operatorname{div}_{s}\left[\mathbb{P}_{\nu}\left(\nabla_{s} \mathbf{u}\right)\right]=\sum_{l=0}^{\infty} r^{-2}\left[X_{l} \mathbf{\Psi}_{l}-2 X_{l} \mathbf{Y}_{l}-(\tilde{l}-1) \Theta_{l} \mathbf{\Psi}_{l}+\tilde{l} \Theta_{l} \mathbf{Y}_{l}\right] \\
& =-\sum_{l=0}^{\infty}\left[\mathbf{Y}_{l}, \mathbf{\Psi}_{l}\right]\left[\begin{array}{cc}
2 & -\tilde{l} \\
-1 & \tilde{l}-1
\end{array}\right]\left[\begin{array}{c}
X_{l} \\
\Theta_{l}
\end{array}\right], \\
& \operatorname{div}_{s}\left[\left(\nabla_{s} \cdot \mathbf{u}\right) \mathbf{I}_{\nu}\right]=\sum_{l=0}^{\infty}\left[2 X_{l}-\tilde{l}_{l}\right] r^{-1} \operatorname{div}_{s}\left(Y_{l} \mathbf{I}_{\nu}\right)=r^{-2} \sum_{l=0}^{\infty}\left[2 X_{l}-\tilde{l}_{l}\right]\left(-2 \mathbf{Y}_{l}+\mathbf{\Psi}_{l}\right) \\
& =-\sum_{l=0}^{\infty} r^{-2}\left[\mathbf{Y}_{l}, \mathbf{\Psi}_{l}\right]\left[\begin{array}{cc}
4 & -2 \tilde{l} \\
-2 & \tilde{l}
\end{array}\right]\left[\begin{array}{c}
X_{l} \\
\Theta_{l}
\end{array}\right], \\
& \operatorname{div}_{s}\left(\nabla_{s} \mathbf{u}\right)=\sum_{l=0}^{\infty} r^{-2}\left[2 X_{l} \mathbf{\Psi}_{l}-(2+\tilde{l}) X_{l} \mathbf{Y}_{l}-\tilde{l} \Theta_{l} \mathbf{\Psi}_{l}+2 \tilde{l} \Theta_{l} \mathbf{Y}_{l}\right] \\
& =-\sum_{l=0}^{\infty} r^{-2}\left[\mathbf{Y}_{l}, \mathbf{\Psi}_{l}\right]\left[\begin{array}{cc}
2+\tilde{l} & -2 \tilde{l} \\
-2 & \tilde{l}
\end{array}\right]\left[\begin{array}{l}
X_{l} \\
\Theta_{l}
\end{array}\right] \text {. }
\end{aligned}
$$

Moreover, by the divergence theorem or direct calculation and (A.2) we find that $\left(\phi_{l}=\right.$ 
$\left.\left[X_{l}, \Theta_{l}\right]\right)$

$$
\begin{aligned}
& \int_{\partial B_{r}}\left|\mathbb{P}_{\nu}\left(\nabla_{s} \mathbf{u}\right)\right|^{2} d a=\sum_{l=0}^{\infty} \boldsymbol{\phi}_{l} \cdot \mathbf{S}_{l}^{a} \phi_{l}, \quad \int_{\partial B_{r}}\left|\nabla_{s} \cdot \mathbf{u}\right|^{2} d a=\sum_{l=0}^{\infty} \phi_{l} \cdot \mathbf{S}_{l}^{b} \phi_{l}, \\
& \int_{\partial B_{r}}\left|\nabla_{s} \mathbf{u}\right|^{2} d a=\sum_{l=0}^{\infty} \phi_{l} \cdot \mathbf{S}_{l}^{c} \phi_{l}, \quad \int_{\partial B_{r}} \operatorname{det}\left(\mathbb{P}_{\nu} \nabla_{s} \mathbf{u}\right) d a=\sum_{l=0}^{\infty} \boldsymbol{\phi}_{l} \cdot \mathbf{S}_{l}^{d} \boldsymbol{\phi}_{l}, \\
& \int_{\partial B_{r}}\left[u_{\nu} \nabla_{s} \cdot \mathbf{u}-\boldsymbol{\nu} \cdot\left(\nabla_{s} \mathbf{u}\right) \mathbf{u}_{s}\right] d a=\sum_{l=0}^{\infty} \phi_{l} \cdot \mathbf{S}_{l}^{e} \boldsymbol{\phi}_{l},
\end{aligned}
$$

where the $2 \times 2$ symmetric matrices are given by

$$
\begin{aligned}
\mathbf{S}_{l}^{a}=\left[\begin{array}{cc}
2 & -\tilde{l} \\
-\tilde{l} & \tilde{l}^{2}-\tilde{l}
\end{array}\right], & \mathbf{S}_{l}^{b}=\left[\begin{array}{cc}
4 & -2 \tilde{l} \\
-2 \tilde{l} & \tilde{l}^{2}
\end{array}\right], \quad \mathbf{S}_{l}^{c}=\left[\begin{array}{cc}
2+\tilde{l} & -2 \tilde{l} \\
-2 \tilde{l} & \tilde{l}^{2}
\end{array}\right], \\
\mathbf{S}_{l}^{d}=\frac{1}{2}\left[\begin{array}{cc}
2 & -\tilde{l} \\
-\tilde{l} & \tilde{l}
\end{array}\right], & \mathbf{S}_{l}^{e}=2 \mathbf{S}_{l}^{d} .
\end{aligned}
$$

Finally, for the linearized Helfrich model discussed in Remark 6, (29), we have that $\left(c_{0}=\right.$ $1 / R)$

$$
\int_{\partial B_{R}}\left(\frac{1}{2} \Delta_{s} u_{\nu}+c_{0}^{2} u_{\nu}\right)^{2} d a=\sum_{l=0}^{\infty} \frac{(1-\tilde{l} / 2)^{2}}{R^{2}} X_{l}^{2} .
$$

B. Dirichlet-to-Neumann maps of Stokes flows inside or outside a sphere

To calculate the strain energy or the rate of energy dissipation in the exterior and interior of the spherical membrane $\partial \Omega_{0}$, we consider the Stokes flow problem or equilibrium problem for an incompressible elastic body for $(\mathbf{v}, p)$ :

$$
\nabla \cdot \mathbf{v}=0, \quad \Delta \mathbf{v}-\nabla p=0 \quad \text { in } \Omega_{0} \cup \bar{\Omega}_{0}^{C} .
$$

For axis-symmetric problems, we write the solutions in vector spherical harmonics as

$$
\mathbf{v}(\mathbf{x})=\sum_{l=0}^{\infty} v_{l}^{r}(r) \mathbf{Y}_{l}+v_{l}^{\theta}(r) \mathbf{\Psi}_{l}, \quad p(\mathbf{x})=\sum_{l=0}^{\infty} p_{l}(r) Y_{l}(\theta) .
$$

For any multipole field we find that (c.f., (A.3))

$$
\left\{\begin{array}{l}
\nabla\left(f(r) \mathbf{Y}_{l}\right)=\hat{\mathbf{r}} \otimes \hat{\mathbf{r}} f^{\prime} Y_{l}+f \hat{\mathbf{r}} \otimes \nabla Y_{l}+f / r Y_{l}(\mathbf{I}-\hat{\mathbf{r}} \otimes \hat{\mathbf{r}}), \\
\nabla\left(f(r) \mathbf{\Psi}_{l}\right)=r f \nabla \nabla Y_{l}+\nabla Y_{l} \otimes \hat{\mathbf{r}}(r f)^{\prime},
\end{array}\right.
$$


and that

$$
\left\{\begin{array}{lll}
\nabla \cdot\left(f(r) \mathbf{Y}_{l}\right)=\left(\frac{\mathrm{d} f}{\mathrm{~d} r}+\frac{2}{r} f\right) Y_{l}, & & \nabla \times\left(f(r) \mathbf{Y}_{l}\right)=-\frac{1}{r} f \boldsymbol{\Phi}_{l}, \\
\nabla \cdot\left(f(r) \mathbf{\Psi}_{l}\right)=-\frac{\tilde{l}}{r} f Y_{l}, & & \nabla \times\left(f(r) \mathbf{\Psi}_{l}\right)=\left(\mathcal{L}_{r} f\right) \boldsymbol{\Phi}_{l}, \\
\nabla \cdot\left(f(r) \boldsymbol{\Phi}_{l}\right)=0, & \nabla \times\left(f(r) \boldsymbol{\Phi}_{l}\right)=-\frac{\tilde{l}}{r} f \mathbf{Y}_{l}-\left(\mathcal{L}_{r} f\right) \mathbf{\Psi}_{l} .
\end{array}\right.
$$

From (B.13)-(B.14), we have the following identities:

$$
\begin{aligned}
& \nabla p=\sum\left(\frac{\mathrm{d} p_{l}}{\mathrm{~d} r} \mathbf{Y}_{l}+\frac{p_{l}}{r} \mathbf{\Psi}_{l}\right), \\
& \nabla \times \mathbf{v}=-\sum_{l=0}^{\infty}\left[\frac{v_{l}^{r}}{r}-\left(\mathcal{L}_{r} v_{l}^{\theta}\right)\right] \mathbf{\Phi}_{l}, \quad \nabla \cdot \mathbf{v}=\sum_{l=0}^{\infty}\left[\left(\frac{d}{d r}+\frac{2}{r}\right) v_{l}^{r}-\frac{\tilde{l}}{r} v_{l}^{\theta}\right] Y_{l}, \\
& \nabla \times \nabla \times \mathbf{v}=\sum_{l=0}^{\infty}\left\{\frac{\tilde{l}}{r}\left[\frac{v_{l}^{r}}{r}-\left(\mathcal{L}_{r} v_{l}^{\theta}\right)\right] \mathbf{Y}_{l}+\mathcal{L}_{r}\left[\frac{v_{l}^{r}}{r}-\left(\mathcal{L}_{r} v_{l}^{\theta}\right)\right] \mathbf{\Psi}_{l}\right\} .
\end{aligned}
$$

If $\nabla \cdot \mathbf{v}=0$, we immediately find that

$$
\Delta \mathbf{v}=-\nabla \times(\nabla \times \mathbf{v})=\frac{\tilde{l}}{r}\left[-\frac{v_{l}^{r}}{r}+\left(\mathcal{L}_{r} v_{l}^{\theta}\right)\right] \mathbf{Y}_{l}+\left(\mathcal{L}_{r}^{2} v_{l}^{\theta}-\mathcal{L}_{r} \frac{v_{l}^{r}}{r}\right) \mathbf{\Psi}_{l},
$$

By (B.15) and (B.16), we find that (B.12) implies that for $r<R$ and $r>R$,

$$
\left\{\begin{array}{l}
\left(\frac{\partial}{\partial r}+\frac{2}{r}\right) v_{l}^{r}-\frac{\tilde{l}}{r} v_{l}^{\theta}=0, \\
\frac{\tilde{l}}{r}\left[-\frac{v_{l}^{r}}{r}+\left(\mathcal{L}_{r} v_{l}^{\theta}\right)\right]-\partial_{r} p_{l}=0, \\
\mathcal{L}_{r}^{2} v_{l}^{\theta}-\mathcal{L}_{r} \frac{v_{l}^{r}}{r}-p_{l} / r=0 .
\end{array}\right.
$$

General solutions to the above equation can be found by inserting trial solutions of for$\mathrm{m}\left(v_{l}^{r}, v_{l}^{\theta}, p_{l}\right) \propto\left(r^{\nu}, r^{\nu}, r^{\nu-1}\right)$ into above equations, yielding an algebraic equation for the exponent $\nu$ :

$$
(\nu+1)(\nu+2)-\tilde{l}-\frac{(\nu-1) \nu(\nu+1)(\nu+2)}{\tilde{l}}+(\nu-1) \nu=0 .
$$

The roots of the above equations are given by $\nu=-l-2,-l, l-1, l+1$, and henceforth, the general solutions to (B.17) in the exterior and interior of the ball $\Omega_{0}$ can be written as $(l \geq 1)$

$$
\left(v_{l}^{r}, v_{l}^{\theta}, p_{l}\right)= \begin{cases}C_{1}^{\mathrm{in}}\left(r^{l-1}, \frac{1}{l} r^{l-1}, 0\right)+C_{2}^{\mathrm{in}}\left(r^{l+1}, \frac{l+3}{l} r^{l+1}, \frac{4 l+6}{l} r^{l}\right) & \text { if } r<R, \\ C_{1}^{\mathrm{ex}}\left(r^{-l-2},-\frac{1}{l+1} r^{-l-2}, 0\right)+C_{2}^{\mathrm{ex}}\left(r^{-l}, \frac{-l+2}{i} r^{-l}, \frac{4 l-2}{l+1} r^{-l-1}\right) & \text { if } r>R,\end{cases}
$$


where $C_{1}^{\text {in }}, C_{2}^{\text {in }}, C_{1}^{\text {ex }}, C_{2}^{\text {ex }}$ are arbitrary constants. By (B.13), we find that at the spherical surface $\partial B_{R}$ the traction is given by (recall that $\boldsymbol{\sigma}=-p \mathbf{I}+\nabla \mathbf{v}+(\nabla \mathbf{v})^{T}$ and $\boldsymbol{\nu}=\hat{\mathbf{r}}$ )

$$
\boldsymbol{\sigma} \boldsymbol{\nu}=-\sum_{l=0}^{\infty} p_{l} \mathbf{Y}_{l}+\sum_{l=0}^{\infty}\left[2 \frac{d v_{l}^{r}}{d r} \mathbf{Y}_{l}+\left(\frac{v_{l}^{r}}{r}+\frac{d v_{l}^{\theta}}{d r}-\frac{v_{l}^{\theta}}{r}\right) \mathbf{\Psi}_{l}\right]=: \sum_{l=0}^{\infty}\left(\sigma_{l}^{r r} \mathbf{Y}_{l}+\sigma_{l}^{r \theta} \mathbf{\Psi}_{l}\right)
$$

where

$$
\sigma_{l}^{r r}=-p_{l}+2 \frac{d v_{l}^{r}}{d r}, \quad \sigma_{l}^{r \theta}=\frac{v_{l}^{r}}{r}+\frac{d v_{l}^{\theta}}{d r}-\frac{v_{l}^{\theta}}{r}
$$

For given boundary values of $\left(v_{l}^{r}, v_{l}^{\theta}\right)$ at $r=R$,

$$
\left.\left(v_{l}^{r}, v_{l}^{\theta}\right)\right|_{r=R}=\left(v_{l}^{r *}, v_{l}^{\theta *}\right),
$$

we can determine the unknown constants $C_{1}^{\text {in }}, C_{2}^{\text {in }}, C_{1}^{\text {ex }}, C_{2}^{\text {ex }}$ by (B.18), and then by (B.20) determines a linear relationship between the interior traction $\left.\left(\sigma_{l}^{r r}, \sigma_{l}^{r \theta}\right)\right|_{r=R-}$ (resp. exterior traction $\left.\left.\left(\sigma_{l}^{r r}, \sigma_{l}^{r \theta}\right)\right|_{r=R+}\right)$ and the given boundary values $\left(v_{l}^{r *}, v_{l}^{\theta *}\right)$. The results are as follows:

$$
\begin{aligned}
& {\left.\left[\begin{array}{l}
\sigma_{l}^{r r} \\
\sigma_{l}^{r \theta}
\end{array}\right]\right|_{r=R-}=\mathbf{T}_{l}^{\text {in }}\left[\begin{array}{l}
v_{l}^{r *} \\
v_{l}^{\theta *}
\end{array}\right], \quad \mathbf{T}_{l}^{\text {in }}=\frac{1}{R}\left[\begin{array}{cc}
1+\frac{3}{l}+2 l & -3(1+l) \\
-\frac{3}{l} & 1+2 l
\end{array}\right],} \\
& {\left.\left[\begin{array}{l}
\sigma_{l}^{r r} \\
\sigma_{l}^{r \theta}
\end{array}\right]\right|_{r=R+}=-\mathbf{T}_{l}^{\mathrm{ex}}\left[\begin{array}{c}
v_{l}^{r *} \\
v_{l}^{\theta *}
\end{array}\right], \quad \mathbf{T}_{l}^{\mathrm{ex}}=\frac{1}{R}\left[\begin{array}{cc}
\frac{2 l^{2}+3 l+4}{l+1} & -3 l \\
-\frac{3}{l+1} & 2 l+1
\end{array}\right] .}
\end{aligned}
$$

In other words, the Dirichlet-Neumann map defined by (40) and (41) can be written as

$$
\begin{gathered}
\left.\boldsymbol{\sigma}^{\mathrm{in}} \boldsymbol{\nu}\right|_{r=R-}=\mathrm{DN}^{\mathrm{in}} \mathbf{v}=\sum_{l=0}^{\infty}\left[\mathbf{Y}_{l}, \mathbf{\Psi}_{l}\right] \mathbf{T}_{l}^{\mathrm{in}}\left[\begin{array}{c}
v_{l}^{r *} \\
v_{l}^{\theta *}
\end{array}\right], \\
-\left.\boldsymbol{\sigma}^{\mathrm{ex}} \boldsymbol{\nu}\right|_{r=R+}=\mathrm{DN}^{\mathrm{ex}} \mathbf{v}=\sum_{l=0}^{\infty}\left[\mathbf{Y}_{l}, \mathbf{\Psi}_{l}\right] \mathbf{T}_{l}^{\mathrm{ex}}\left[\begin{array}{c}
v_{l}^{r *} \\
v_{l}^{\theta *}
\end{array}\right] .
\end{gathered}
$$

Also, the energy dissipated in the interior (resp. exterior) flow is given by

$$
\begin{aligned}
& D^{\mathrm{in}}[\mathbf{v}]=\int_{B_{R}}|\nabla \mathbf{v}|^{2} d v=\int_{\partial B_{R}} \mathbf{v} \cdot \boldsymbol{\sigma}^{\mathrm{in}} \boldsymbol{\nu} d a=R \sum_{l=0}^{\infty}\left[v_{l}^{r *} v_{l}^{\theta *}\right] \mathbf{D}_{l}^{\mathrm{in}}\left[\begin{array}{c}
v_{l}^{r *} \\
v_{l}^{\theta *}
\end{array}\right], \\
& D^{\mathrm{ex}}[\mathbf{v}]=\int_{\mathbb{R}^{3} \backslash B_{R}}|\nabla \mathbf{v}|^{2} d v=\int_{\partial B_{R}}-\mathbf{v} \cdot \boldsymbol{\sigma}^{\mathrm{ex}} \boldsymbol{\nu} d a=R \sum_{l=0}^{\infty}\left[v_{l}^{r *} v_{l}^{\theta *}\right] \mathbf{D}_{l}^{\mathrm{ex}}\left[\begin{array}{c}
v_{l}^{r *} \\
v_{l}^{\theta *}
\end{array}\right],
\end{aligned}
$$

where

$$
\mathbf{D}_{l}^{\text {in }}=\left[\begin{array}{cc}
1+\frac{3}{l}+2 l & -3(1+l) \\
-3(l+1) & l(l+1)(2 l+1)
\end{array}\right], \quad \mathbf{D}_{l}^{\mathrm{ex}}=\left[\begin{array}{cc}
\frac{2 l^{2}+3 l+4}{l+1} & -3 l \\
-3 l & l(l+1)(2 l+1)
\end{array}\right] .
$$


C. A numerical algorithm for solving the contact problems

Our numerical method for solving the contact problems is based on the variational inequality (84). Let $2 l_{\max }$ be the maximum spherical harmonics and $\theta_{i}=\frac{i-1}{K} \theta_{M}(i=$ $1,2, \cdots, K+1)$ be discrete points on the interval $\left[0, \theta_{M}\right]$. We shall set $\theta_{M}=\sin ^{-1}\left(a_{0} / R\right)$ for a flat-headed indenter of radius $a_{0}$, and $\theta_{M}=\frac{\pi}{2}$ for rigid plates.

We recall that the elastic energy of the system is given by

$$
\begin{aligned}
& E\left[\phi_{l}\right]=\sum_{l=2}^{2 l_{\max }} \frac{1}{2} \phi_{l} \cdot \mathbf{S}_{l} \phi_{l}, \quad \phi_{l}=\left(X_{l}, \Theta_{l}\right), \\
& \left(u^{r}(R, \theta), u^{\theta}(R, \theta)\right)=\sum_{l=2}^{2 l_{\max }}\left(X_{l} Y_{l}(\theta), \Theta_{l} Y_{l}^{\prime}(\theta)\right) .
\end{aligned}
$$

Enforcing the constraint (84) only on these discrete points $\theta_{i}$, we obtain

$$
\sum_{l=2}^{2 l_{\max }} X_{l} Y_{l}\left(\theta_{i}\right) \cos \theta_{i}-\sum_{l=2}^{2 l_{\max }} \Theta_{l} Y_{l}^{\prime}\left(\theta_{i}\right) \sin \theta_{i} \leq z_{\text {in }}+h\left(\theta_{i}\right)-R \cos \theta_{i}(i=1, \cdots, K+1),(
$$

which implies $(K+1)$-linear inequality constraints on the unknown $\left(X_{l}, \Theta_{l}\right)\left(l=2,4, \cdots, 2 l_{\max }\right)$. Then in terms of the unknown mode amplitude $\left(X_{l}, \Theta_{l}\right)$, the discretized variational inequality problem (84) can be expressed as

$$
\min \left\{E\left[\phi_{l}\right]=\sum_{l=2}^{2 l_{\max }} \frac{1}{2} \phi_{l} \cdot \mathbf{S}_{l} \phi_{l}: \phi_{l}\left(l=2,4, \cdots, 2 l_{\max }\right) \text { satisfy (C.27) }\right\} .
$$

The above minimization problem can be identified as a quadratic programming problem with $(K+1)$-linear inequality constraints on the unknown $\left(X_{l}, \Theta_{l}\right)\left(l=2,4, \cdots, 2 l_{\max }\right)$. Solutions can be conveniently found by the quadprog.m program in MATLAB.

Acknowledgement L.L. gratefully acknowledges the support of NSF CMMI-135156, DMS1410273, NSFC-1152800009, and AFOSR-FA9550-16-1-0181.

Emad A-Hassan, William F. Heinz, Matthew D. Antonik, Neill P. DCosta, Soni Nageswaran, Cora-Ann Schoenenberger, and Jan H. Hoh. Relative microelastic mapping of living cells by atomic force microscopy. Biophysical Journal, 74(3):1564 - 1578, 1998.

Thomas E. Angelini, Edouard Hannezo, Xavier Trepat, Manuel Marquez, Jeffrey J. Fredberg, and David A. Weitz. Glass-like dynamics of collective cell migration. Proceedings of the National Academy of Sciences, 108(12):4714-4719, 2011.

Marino Arroyo and Antonio DeSimone. Relaxation dynamics of fluid membranes. Phys. Rev. E, 79:031915, 2009.

G. Bao and S. Suresh. Cell and molecular mechanics of biological materials. Nature Materials, 2:715-725, 2003. 
A. R. Bausch, W. Möller, and E. Sackmann. Measurement of local viscoelasticity and forces in living cells by magnetic tweezers. Biophysical Journal, 76(1):573 - 579, 1999.

D. Bi, J. H. Lopez, J. M. Schwarz, and M. Lisa Manning. A density-independent rigidity transition in biological tissues. Nature Physics, 11:1074-1079, 2015.

A. F. Bower. Applied Mechanics of Solids. CRC Press, 2009.

P.B. Canham. The minimum energy of bending as a possible explanation of the biconcave shape of the human red blood cell. Journal of Theoretical Biology, 26(1):61 - 81, 1970.

S. Chien, K. L. Sung, R. Skalak, S. Usami, and A Tözeren. Biophysical Journal. Theoretical and experimental studies on viscoelastic properties of erythrocyte membrane, 24:463-487, 1978.

M. Dao, C.T. Lim, and S. Suresh. Mechanics of the human red blood cell deformed by optical tweezers. Journal of the Mechanics and Physics of Solids, 51:2259 - 2280, 2003.

A. Diz-Muñoz, D. A. Fletcher, and O.D. Weiner. Use the force: membrane tension as an organizer of cell shape and motility. Trends in Cell Biology, 23:47 - 53, 2013.

E. Evans and D. Needham. Physical properties of surfactant bilayer membranes:thermal transitions, elasticity, rigidity, cohesion, and colloidal interactions. J. Phys. Chem, 91: 4219, 1987.

Ben Fabry, Geoffrey N. Maksym, James P. Butler, Michael Glogauer, Daniel Navajas, and Jeffrey J. Fredberg. Scaling the microrheology of living cells. Phys. Rev. Lett., 87:148102, 2001.

D. A. Fletcher and R. D. Mullins. Cell mechanics and the cytoskeleton. Nature, 463:485-492, 2010 .

G. Forgacs, R. A. Foty, Y. Shafrir, and M. S. Steinberg. Viscoelastic properties of living embryonic tissues: a quantitative study. Biophys. J., 74(5):2227-2234, 1998.

Ramsey A Foty. Tumor cohesion and glioblastoma cell dispersal. Future Oncology, 2013.

Ramsey A. Foty. Measurement of Intercellular Cohesion by Tissue Surface Tensiometry, pages 237-254. Springer New York, New York, NY, 2015.

Ramsey A. Foty, Gabor Forgacs, Cathie M. Pfleger, and Malcolm S. Steinberg. Liquid properties of embryonic tissues: Measurement of interfacial tensions. Phys. Rev. Lett., 72: 2298-2301, 1994.

M. L. Gardel, M. T. Valentine, J. C. Crocker, A. R. Bausch, and D. A. Weitz. Microrheology of entangled f-actin solutions. Phys. Rev. Lett., 91:158302, 2003.

M. L. Gardel, J. H. Shin, F. C. MacKintosh, L. Mahadevan, P. Matsudaira, and D. A. Weitz. Elastic behavior of cross-linked and bundled actin networks. Science, 304(5675): 1301-1305, 2004. 
Sefi Givli, Ha Giang, and Kaushik Bhattacharya. Stability of multicomponent biological membranes. SIAM Journal on Applied Mathematics, 72(2):489-511, 2012.

M.E. Gurtin and A.I. Murdoch. A continuum theory of elastic material surfaces. Arch. Rat. Mech. Anal., 57(4):291-323, 1975.

W. Helfrich. Elastic properties of lipid bilayers theory and possible experiments. Z. Naturforsch., C28:693, 1973.

R. M. Hochmuth. Journal of Biomechanics. Micropipette aspiration of living cells, 33:15 22,2000 .

L.X. Hu and L.P. Liu. Interfacial waves with surface elasticity. J. Appl. Mech, 81:081007, 2014.

Philip Kollmannsberger and Ben Fabry. Linear and nonlinear rheology of living cells. Annu. Rev. Mater. Res., 41:75-97, 2011.

Wolfgang Kühnel. Differential Geometry: Curves - Surfaces - Manifolds. American Mathematical Society, 2nd Ed., 2005.

M. Lisa Manning, Ramsey A. Foty, Malcolm S. Steinberg, and Eva-Maria Schoetz. Coaction of intercellular adhesion and cortical tension specifies tissue surface tension. Proceedings of the National Academy of Sciences, 107(28):12517-12522, 2010.

E. Moeendarbary, L. Valon, M. Fritzsche, A.R. Harris, G.T. Charras, D.A. Moulding, A.J. Thrasher, E. Stride, and L. Mahadevan. The cytoplasm of living cells behaves as a poroelastic material. Nature Materials, 12:253-261, 2013.

Emad Moeendarbary and Andrew R. Harris. Cell mechanics: principles, practices, and prospects. Wiley Interdisciplinary Reviews: Systems Biology and Medicine, 6(5):371-388, 2014.

Mohammad R. K. Mofrad. Rheology of the cytoskeleton. Annu. Rev. Fluid Mech., 41: 433-453, 2009 .

S. Nam, K. H. Hu, M. J. Butte, and O. Chaudhuri. Strain-enhanced stress relaxation impacts nonlinear elasticity in collagen gels. Proc. Natl. Acad. Sci. U.S.A., 113(20):5492-5497, 2016.

YongKeun Park, Catherine A. Best, Kamran Badizadegan, Ramachandra R. Dasari, Michael S. Feld, Tatiana Kuriabova, Mark L. Henle, Alex J. Levine, and Gabriel Popescu. Measurement of red blood cell mechanics during morphological changes. Proceedings of the National Academy of Sciences, 107(15):6731-6736, 2010.

Gabriel Popescu, Takahiro Ikeda, Keisuke Goda, Catherine A. Best-Popescu, Michael Laposata, Suliana Manley, Ramachandra R. Dasari, Kamran Badizadegan, and Michael S. Feld. Optical measurement of cell membrane tension. Phys. Rev. Lett., 97:218101, 2006. 
Juan Ren, Huarong Huang, Yue Liu, Xi Zheng, and Qingze Zou. An atomic force microscope study revealed two mechanisms in the effect of anticancer drugs on rate-dependent youngs modulus of human prostate cancer cells. PLoS ONE, 10(5):1-14, 2015.

M. J. Rosenbluth, W. A. Lam, and D. A. Fletcher. Force microscopy of nonadherent cells: a comparison of leukemia cell deformability. Biophys. J., 90(8):2994-3003, 2006.

U. Seifert. Fluid membranes in hydrodynamic flow fields: Formalism and an application to fluctuating quasispherical vesicles in shear flow. Eur. Phys. J. B, 8(3):405-415, 1999.

Udo Seifert. Configurations of fluid membranes and vesicles. Advances in Physics, 46(1): 13-137, 1997.

Christine Semmrich, Tobias Storz, Jens Glaser, Rudolf Merkel, Andreas R. Bausch, and Klaus Kroy. Glass transition and rheological redundancy in f-actin solutions. Proc. Natl. Acad. Sci. U.S.A., 104(51):20199-20203, 2007.

Stephen Shannon, Connan Vaca, Dongxuan Jia, Ildiko Entersz, Andrew Schaer, Jonathan Carcione, Michael Weaver, Yoav Avidar, Ryan Pettit, Mohan Nair, Atif Khan, and Ramsey A. Foty. Dexamethasone-mediated activation of fibronectin matrix assembly reduces dispersal of primary human glioblastoma cells. PLoS ONE, 10(8):1-26, 082015.

John Sleep, David Wilson, Robert Simmons, and Walter Gratzer. Elasticity of the red cell membrane and its relation to hemolytic disorders: An optical tweezers study. Biophysical Journal, 77(6):3085 - 3095, 1999.

I. Sokolov. Biophysical Journal. Apparent viscosity and cortical tension of blood granulocytes determined by micropipet aspiration, 56:151-160, 1989.

I. Sokolov. Atomic Force Microscopy in Cancer Cell Research. Cancer Nanotechnology (Ed. H. Singh and T. Webster), Ch. 1:1-17, 2007.

D.J. Steigmann, E. Baesu, Jim Belak R.E. Rudd, and M. McElfresh. On the variational theory of cell-membrane equilibria. Interfaces and Free Boundaries, 5(4):357-366, 2003.

J. Stricker, T. Falzone, and M. L. Gardel. Mechanics of the f-actin cytoskeleton. J. Biomech., 43(1):9-14, 2010.

K. Sugimura, P. F. Lenne, and F. Graner. Measuring forces and stresses in situ in living tissues. Development, 143(2):186-196, 2016.

Yubing Sun, Luis G. Villa-Diaz, Raymond H. W. Lam, Weiqiang Chen, Paul H. Krebsbach, and Jianping $\mathrm{Fu}$. Mechanics regulates fate decisions of human embryonic stem cells. PLoS ONE, 7(5):1-7, 052012.

Subra Suresh. Biomechanics and biophysics of cancer cells. Acta Biomaterialia, 3(4):413 438, 2007. 
D.P. Theret, M.J. Levesque, M.M. Sato, R.M. Nerem, and L.T. Wheeler. ASME. J Biomech Eng. The Application of a Homogeneous Half-Space Model in the Analysis of Endothelial Cell Micropipette Measurements, 110:190-199, 1988.

Nikhil Walani and Ashutosh Agrawal. Stability of lipid membranes. Mathematics and Mechanics of Solids, 2015.

N Wang, JP Butler, and DE Ingber. Mechanotransduction across the cell surface and through the cytoskeleton. Science, 260(5111):1124-1127, 1993.

Miao Yu, Rafael B. Lira, Karin A. Riske, Rumiana Dimova, and Hao Lin. Ellipsoidal relaxation of deformed vesicles. Phys. Rev. Lett., 115:128303, 2015.

J. Zhang, J. D. Zahn, and H. Lin. Transient solution for droplet deformation under electric fields. Phys. Rev. E, 87:043008, 2013. 